\title{
RGB-D Data-Based Action Recognition: A Review
}

\author{
Muhammad Bilal Shaikh *,+(i) and Douglas Chai ${ }^{+}$(D) \\ School of Engineering, Edith Cowan University, Perth, WA 6027, Australia; d.chai@ecu.edu.au \\ * Correspondence: m.shaikh@ecu.edu.au \\ † These authors contributed equally to this work.
}

check for

updates

Citation: Shaikh, M.B.; Chai, D. RGB-D Data-Based Action

Recognition: A Review. Sensors 2021,

21, 4246. https://doi.org/

$10.3390 / \mathrm{s} 21124246$

Academic Editor: Xue-Bo Jin

Received: 28 March 2021

Accepted: 9 June 2021

Published: 21 June 2021

Publisher's Note: MDPI stays neutral with regard to jurisdictional claims in published maps and institutional affiliations.

\begin{abstract}
Classification of human actions is an ongoing research problem in computer vision. This review is aimed to scope current literature on data fusion and action recognition techniques and to identify gaps and future research direction. Success in producing cost-effective and portable vision-based sensors has dramatically increased the number and size of datasets. The increase in the number of action recognition datasets intersects with advances in deep learning architectures and computational support, both of which offer significant research opportunities. Naturally, each action-data modality—such as RGB, depth, skeleton, and infrared (IR) — has distinct characteristics; therefore, it is important to exploit the value of each modality for better action recognition. In this paper, we focus solely on data fusion and recognition techniques in the context of vision with an RGB-D perspective. We conclude by discussing research challenges, emerging trends, and possible future research directions.
\end{abstract}

Keywords: action recognition; deep learning; data fusion; RGB-D

\section{Introduction}

Human action recognition (HAR) has recently gained increasing attention from computer vision researchers with applications in robot vision, multimedia content search, video surveillance, and motion tracking systems. The recent developments in artificial intelligence have stimulated computer vision researchers to investigate problems in recognizing actions. Coupled with the vast amount of digital data available today, the rise of deep learning has resulted in a dramatic increase in computing resources and offers attractive opportunities for designing efficient action recognition systems.

The development of low-cost sensors such as Microsoft Kinect [1], Intel RealSense [2], and Orbbec [3] has sparked further research into action recognition. These sensors collect data in various modalities such as RGB video, depth, skeleton, and IR. All these modalities have their own characteristics that can help answer challenges related to action data and provide potential opportunities for computer vision researchers to examine vision data from different perspectives.

Herath et al. [4] have defined action as "the most elementary human-surrounding interaction with a meaning". HAR is the process of labeling the actions performed by humans within a given sequence of images, where it becomes the classification of goals of a human agent in a series of image frames. Action recognition typically aims to discover the class of short, segmented, atomic action. However, action detection (or event detection, or annotation) algorithms reason not only about whether an action occurs somewhere in a video, but also on the temporal extent of when it occurs. Due to their multifaceted nature, some of these approaches refer to action recognition as plan recognition, goal recognition, intent recognition, behavior recognition, location estimation, event recognition, action recognition, and interaction recognition. Some of the terms referenced in the literature in relation to action are defined in Table 1. 
Table 1. Terms related to action recognition.

\begin{tabular}{|c|c|}
\hline Term & Definition \\
\hline Gesture, Mime, Sign & $\begin{array}{l}\text { Basic movement or positioning of the hand, arm, body, } \\
\text { or head that communicates an idea, emotion, etc. }\end{array}$ \\
\hline Action, Event & $\begin{array}{l}\text { A type of motion performed by a single person during } \\
\text { short time period and involves multiple body parts. }\end{array}$ \\
\hline Activity & Composed of a sequence of actions. \\
\hline Interaction & $\begin{array}{l}\text { A type of motion performed by two actors; one actor is } \\
\text { human while the other may be human or an object. }\end{array}$ \\
\hline Unimodal, Single-mode & Having or involving one mode. \\
\hline Multimodal, Multi-type, Multi-format & Different types of data acquired through sensors. \\
\hline Fusion, Mixture, Combination & A process for combining different types of sensor data. \\
\hline RGB-D & $\begin{array}{l}\text { Per-pixel depth information aligned with corresponding } \\
\text { image pixels. }\end{array}$ \\
\hline
\end{tabular}

Early research on Human Action Recognition was dominated by the analysis of still images or videos [5-10], localizing the actor in a video spatio-temporally using bounding boxes, temporal extent, and a spatio-temporal cuboid which contains a particular action. Action recognition remains challenging due to problems posed by background clutter, partial occlusion, viewpoint, lighting changes, execution rate, and biometric variation. These challenges remain even with the application of current deep learning-based approaches $[4,11]$. Understanding information from images is a challenging process that has engaged thousands of researchers for over four decades and studies are still far from developing a general-purpose machine that can "see" [12].

Human Action Recognition has many applications, including the automated annotation of user videos, indexing and retrieving user videos, automated surveillance, monitoring elderly patients using specially adapted cameras, robot operations, and live blogging of actions. In recent times, the availability of massive amounts of video data has provided significance to the understanding of video data (through a sequence of images) with the possibility of solving problems such as scene identification, searching through video content, and interaction recognition through video scenes [13].

Several survey papers [14-25] have discussed action recognition from different perspectives. This survey is unique as it focuses action recognition methods on various RGB-D modalities. RGB-D, which stands for Red Green Blue-Depth, provides depth information associated with corresponding RGB data. However, some relatable surveys, such as those in [26-29], study from a single modality perspective or compare the characteristics of different datasets. According to our knowledge, there is no review with a focus specifically on data fusion and vision-based action recognition in the context of RGB-D data. The literature was searched by using the keywords "action recognition" over the period from 2010 to 2020. In vision-based action recognition, classification techniques take distinctive characteristics from each modality and apply computer vision methods. This paper offers computer vision researchers a set of potential opportunities to explore vision data by exploiting the natural characteristics of different modalities.

A novel contribution of this review is the focus on RGB-D data-based action recognition using deep learning-based methods. Moreover, this work distinguishes itself from other studies through the following contributions.

1. Review of state-of-the-art action recognition techniques on common RGB-D datasets that will provide readers with an overview of recent developments in action recognition.

2. Analysis of current methods from a perspective of multimodality and hybrid classification methods.

3. Intuitive categorization and analysis of recent and advanced classical machine learningbased and deep learning-based techniques.

4. Discussion of the challenges of data fusion and action recognition and potentials future research directions. 
The remainder of this paper is organized as follows. Section 2 discusses RGB-D modality and data acquisition. Sections 3 and 4 review the classical machine learningbased methods and deep learning-based methods, respectively. Section 5 discusses the use of different data fusion techniques used in HAR. Section 6 gives a summary of applications of state-of-the-art RGB-D methods in different scenarios. Section 7 outlines different challenges in data fusion and action recognition techniques, and discusses the future research directions. Finally, Section 8 concludes the review. The hierarchical structure of this paper is shown in Figure 1.

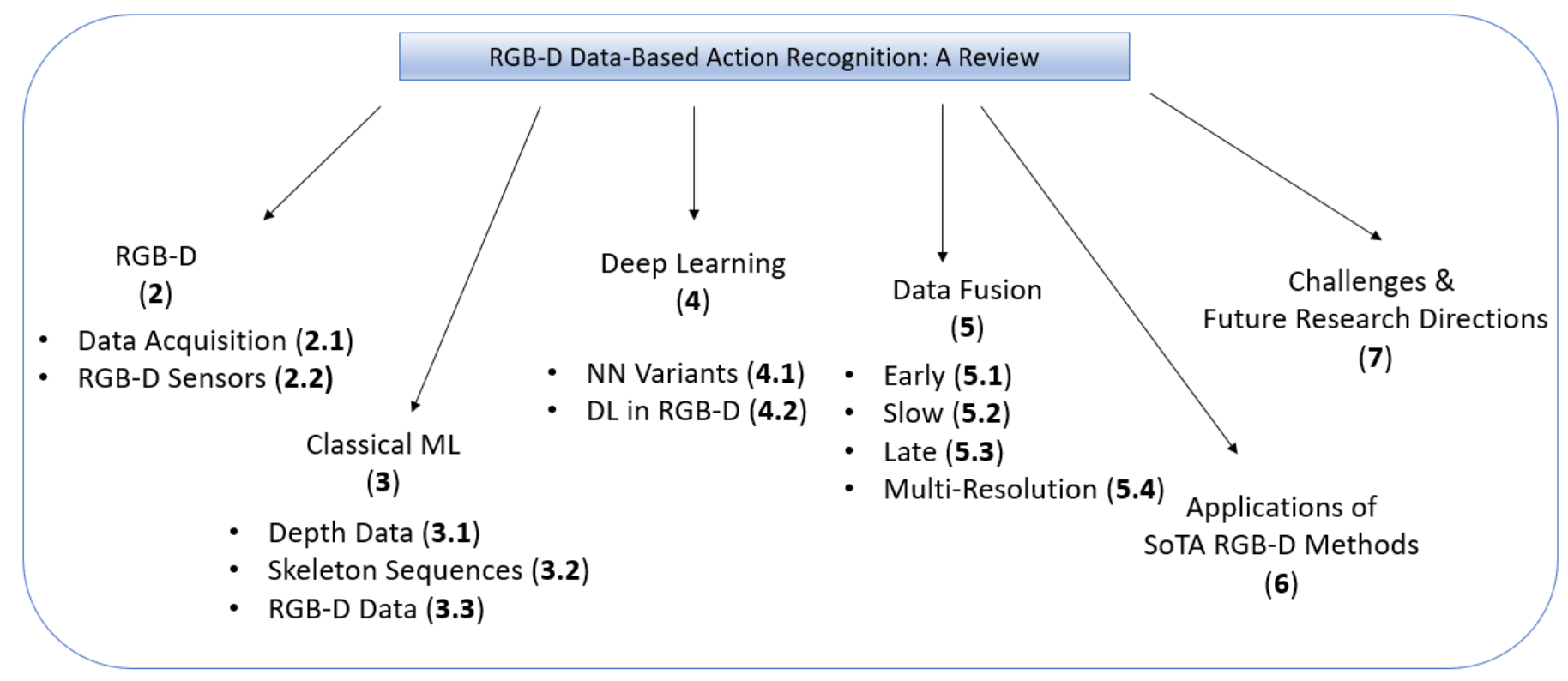

Figure 1. Structure of this paper. Numbers in brackets refer to the section numbering.

\section{RGB-D}

RGB-D generally refers to Red, Green, Blue plus Depth data captured by RGB-D sensors. An RGB-D image provides a per-pixel depth information aligned with corresponding image pixels. An image formed through depth information is an image channel in which each pixel relates to a distance between the image plane and the corresponding object in the RGB image. The addition of depth information to conventional RGB image helps improve the accuracy and the denseness of the data. An example of data captured by an RGB-D sensor is shown in Figure 2. RGB-D data acquisition and different consumer preferred sensors will be discussed in following subsections.

\subsection{RGB-D Data Acquisition}

Acquisition of depth information is mainly based on triangulation and Time-of-Flight (ToF) techniques. The former technique may be implemented passively using stereovision, which retrieves depth information by capturing the same scene from different point of views. Stereovision emulates a human vision principle where depth is computed as a disparity between images taken from different viewpoints. This may require knowledge of the geometry of cameras and calibration needs to be performed for each change in system configuration. An active approach relies on structured light, which uses an IR light pattern onto the scene to estimate disparity through varying object's depth. In addition to this, ToF and Light Detection and Ranging (LiDAR) scanners measure the time that light takes to hit an object's surface and return to the detector. LiDAR uses mechanical components to its surrounding. However, ToF performs distance computation using integrated circuits. Chen et al. [22] and others [30] have briefly surveyed depth data acquisition in RGB-D sensors. 


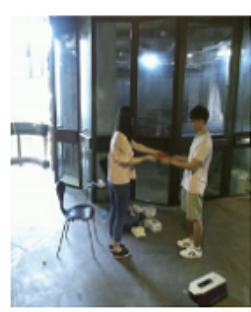

(a)

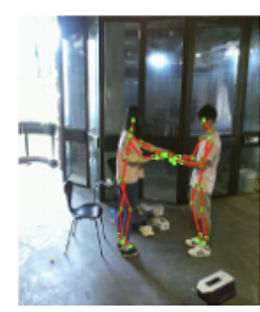

(b)

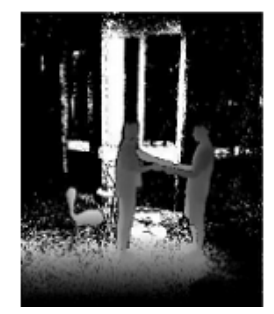

(c)

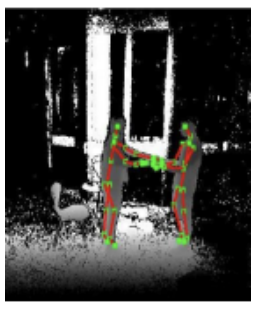

(d)

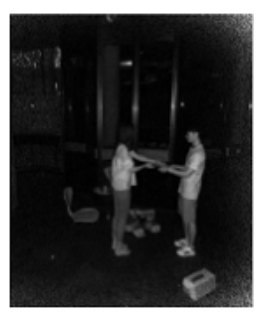

(e)

Figure 2. Example data captured by an RGB-D sensor as taken from the NTU RGB-D dataset [31] in (a) RGB, (b) RGB + Skeleton Joints, (c) Depth, (d) Depth + Skeleton Joints, and (e) IR modalities.

\subsection{RGB-D Sensors}

Most of the consumer RGB-D sensors rely on structured light or ToF approaches. Such RGB-D sensors possess noise and data distortions, which are tackled by specifically designed algorithms. Nevertheless, ToF provides a better depth resolution than the others, which is about a few millimeters. Moreover, structured light systems are not beneficial in outdoor scenarios because solar light strongly affects IR cameras. HAR tasks that do not require very high depth resolution and precision have been easily implemented using both structured light sensors and ToF devices. Such devices represented a very good compromise between cost, performance, and usability, and allowed implementation of unobtrusive and privacy-preserving solutions. Some consumer-preferred RGB-D sensors are outlined in the following subsections.

\subsubsection{Microsoft ${ }^{\circledR}$ Kinect $^{\mathrm{TM}}$ Sensors}

Microsoft released the Kinect RGB-D sensor, a low-cost but high-resolution tool that could be easily interfaced to a computer, and whose signals could be easily manipulated through common academic practices. The Kinect sensor V1 uses structured light, and Kinect V2 is based on ToF. The latter exhibits less software complexity but requires fast hardware, such as pulse width modulation (PWM) drivers. The Kinect technology pushed the development of depth-based algorithms and processing approaches. Kinect has been discontinued, but alternative sensors are available in the market. Azure Kinect is a recent spatial computing developer kit with computer vision and speech models, and a range of development interfaces that can be connected to Azure cognitive services. Azure Kinect is not available for consumers and thus not a replacement of Kinect. Michal et al. [32] presented a comprehensive evaluation of Azure Kinect and its comparison with both versions of Kinect. Different versions of Kinect Sensor are shown in Figure 3a (from bottom to top-Kinect v1, Kinect v2, and Azure Kinect).

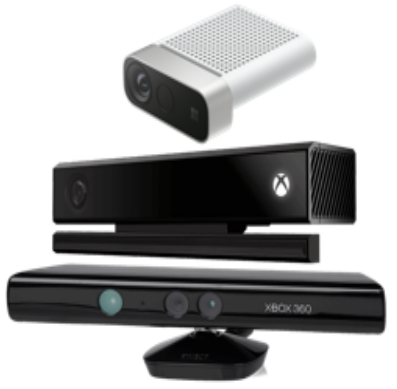

(a)

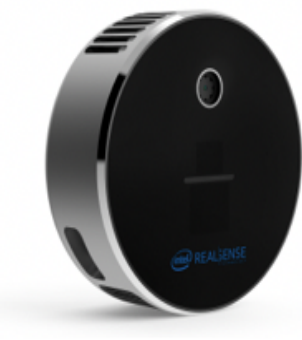

(b)

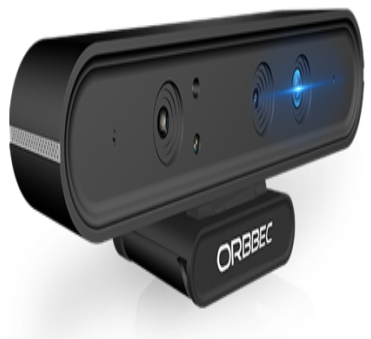

(c)

Figure 3. Various RGB-D sensors: (a) Microsoft Kinect [33-35], (b) Intel RealSense L515 [36], and (c) Orbbec Astra Pro [37].

The Kinect sensor makes the task of capturing RGB-D data easier by sensing the depth dimension of the subject and its environment. It also interprets the movement performed by a subject and transforms it into a format that practitioners can use for new experiments. 
Computer vision researchers have leveraged Kinect's vision technology for performing tasks such as aiding children to overcome autism [38] and for doctors in their operating rooms. Azure Kinect has been released for developers and industries which will potentially transform human-computer interaction in various industries including manufacturing, education [39], healthcare [40], retail [41], transportation [42], and beyond.

\subsubsection{Intel ${ }^{\circledR}$ RealSense ${ }^{\mathrm{TM}}$ Depth Cameras}

Intel RealSense depth cameras encompass a family of stereoscopic and portable RGBD sensors which includes subpixel disparity accuracy, assisted illumination, and performs well even in outdoor settings. Keselman et al. [43] provided a brief overview of Intel RealSense cameras. The R400 family is successor to the R200 family that includes improvements in its stereoscopic matching algorithm and correlation cost function as well as an optimization in design, which enables the R400 family to consume lower power than R200 while operating on the same image resolutions. Intel has divided its RGB-D sensors into different categories which includes stereo depth, LiDAR, coded light, and tracking sensors. The Intel RealSense LiDAR Camera L515 [44] shown in Figure 3b is the smallest highresolution LiDAR depth camera to date. The Intel D400 [45] series uses Active IR stereo technology. The Intel SR [46] series uses coded light technology; however, the recently introduced $\mathrm{L}$ series uses LiDAR technology for acquiring depth information. The $\mathrm{L}$ series has significantly reduced the size of the sensor, which can accelerate the use of RGB-D sensors in HAR.

\subsubsection{Orbbec ${ }^{\circledR}$ Depth Cameras}

Orbbec Astra sensors incorporate processor which replaces traditional cable-based connection to sensor. Similar to Kinect, the Orbbec Astra Pro device as shown in Figure $3 c$ includes an RGB camera, a depth camera, an IR projector, and two microphones. In addition to this, the Orbecc camera-computer package is economical compared to Kinect or RealSense devices. Several SDKs are available including Astra SDK (developed by the manufacturers of the sensor) and OpenNI framework for 3D natural interaction sensors. The use of different sensors in the same problem could affect the accuracy of the process. Coroiu et al. [47] demonstrated safe exchange of Kinect sensor with the Orbbec sensor. According to the experiments, over 16 classifiers demonstrated that choice of sensor does not affect the accuracy. However, seven classifiers produced a drop-in accuracy. Furthermore, calibration algorithms using different RGB-D sensor are compared in [48]. In general, RGB-D sensors exhibit acceptable accuracy, but in some cases, calibration processes are critical to increase the sensor's accuracy and enable it to meet the requirements of such kinds of applications.

\section{Classical Machine Learning-Based Techniques}

Classical machine learning-based action recognition techniques use handcrafted features and can be classified on the basis of RGB data [18], depth data [49,50], skeleton sequences [51], and methods using a combination [52] of these data modalities (as illustrated in Figure 4). Table 2 summarizes the best performing techniques which achieved benchmark accuracies for popular RGB-D datasets in action recognition research. The following subsections will discuss depth-, skeleton-, and RGB-D-based methods.

\subsection{Depth Data-Based Techniques}

Motion changes in the depth maps of the human body are used to represent action. Depth data can be observed as a space-time structure which is extracted from the appearance and motion information to describe human actions. Yang et al. [53] have proposed a supernormal vector feature through a depth map sequence for action representation. Oreifej et al. [49] have proposed an orientation histogram feature of $4 \mathrm{D}$ normal vectors to represent the appearance information of a 3D spatio-temporal depth structure. Rehmani et al. [54] have proposed the idea of the main direction of a depth-curved surface 
where a perspective-independent feature and a principal component histogram are used to represent action. Yang et al. [55] have proposed the Depth Motion Map (DMM) to project spatio-temporal depth structure onto motion history maps. More recent motion history maps are represented by Histogram of Gradients (HoG) features in series to represent actions. Chen et al. [56] have used local binary features instead of HoG features; they [57] also investigated spatio-temporal depth structure from front, side, and upper directions. Miao et al. [58] have considered discrete cosine variation to compress the depth map and represent action through features using transform coefficients.

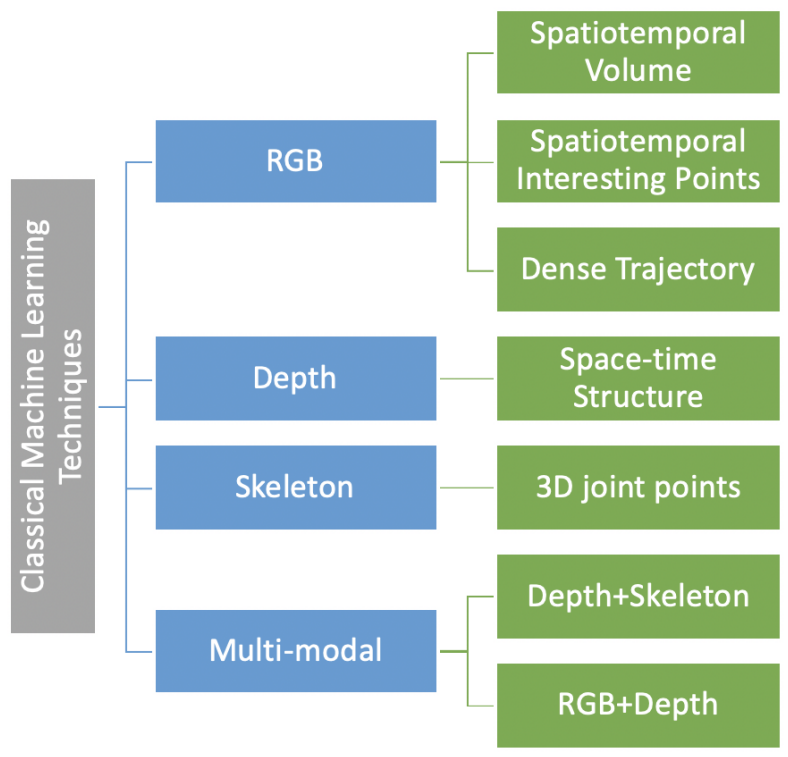

Figure 4. Hierarchy of action recognition techniques based on handcrafted features that use classical machine learning.

\subsection{Skeleton Sequence-Based Techniques}

Changes in position and appearance changes in human joint points between frames are used to describe action. Xia et al. [59] have modeled action through a discrete hidden Markov model. Action features have also been extracted through 3D Histograms of Oriented Displacements (HoD) [60], Accumulation of Motion Energy (AME) function aided with the Eigenjoint-based method [51], and through a longest common sequence algorithm [61] to select high-discriminative power features from the relative motion trajectories of the skeleton.

\subsection{RGB-D Data-Based Techniques}

The research results in $[50,54,62]$ show that depth-based methods achieve better action recognition performance than RGB-based methods. Therefore, some researchers have also tried a fusion of different modalities. Chaaroui et al. [63] have investigated the fusion of skeleton and depth data to overcome problems caused by occlusion and perspective changes in skeleton features. In addition, a sparse regression learning-based method to fuse depth and skeleton features has been proposed by Li et al. [52]. A multi-kernelbased learning method for describing actions has been proposed by Althloothi et al. [64]; they calculated spherical harmonics through depth data and fused this with the spatial information of skeleton joints. Furthermore, RGB and depth data fusion has also been attempted by some researchers. For example, Liu et al. [65] used generic algorithms, Jalal et al. [66] merged spatio-temporal features, and Ni et al. [67] introduced the multi-level fusion of RGB and depth data features. However, to answer the missing modality problem, Kong et al. [68] have proposed a discriminative relational representation learning (DRRL) method. In the absence of a single modality in testing, this method transfers knowledge from training data to substitute the missing modality and achieves better recognition 
performance. The main concern with RGB-D data fusion is that it adds more computational complexity to the action recognition algorithm. Yu et al. [69] have proposed a binary representation for RGB-D data fusion with structure-preserving projections. This approach produced high efficiency and effectiveness on various action recognition benchmarks of RGB-D data. Different challenges associated with RGB-D data fusion techniques are discussed in Section 7.1.

Table 2. Summary of popular action recognition datasets and methods that achieved the best recognition accuracy. Note that PDF stands for probability distribution function, i3D stands for inflated 3D, OF stands for Optical Flow, and GCN stands for Graph Convolutional Networks.

\begin{tabular}{|c|c|c|c|c|c|c|c|c|c|c|c|c|c|c|c|c|c|c|}
\hline Year & Ref. & Methods (Modality) & 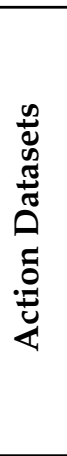 & 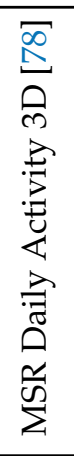 & 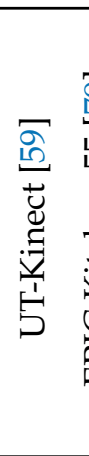 & 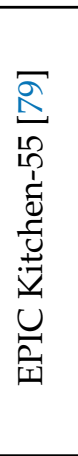 & 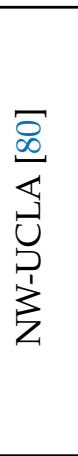 & 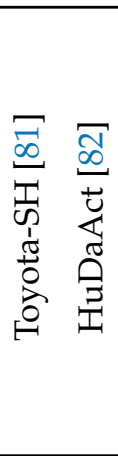 & 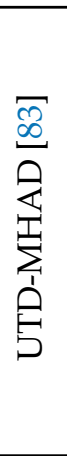 & 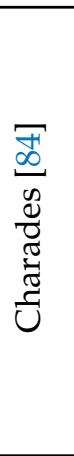 & 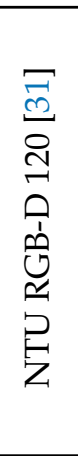 & 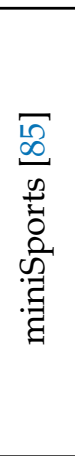 & 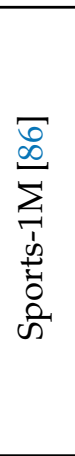 & $\begin{array}{l}\infty \\
\infty \\
\stackrel{\infty}{\infty} \\
\stackrel{\infty}{\sigma} \\
\stackrel{\sigma}{\sigma}\end{array}$ & 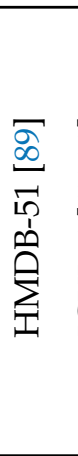 & 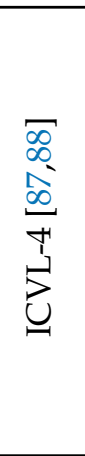 & 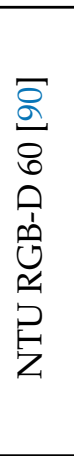 & 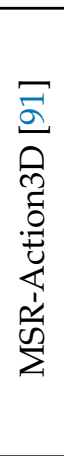 \\
\hline 2012 & [92] & 2D CNN (RGB-D) & & & & & & 89 & & & & & & & & & & \\
\hline 2015 & [93] & DTQ-SVM (RGB-D) & & & 100 & & & & & & & & & & & & & 90 \\
\hline 2017 & [94] & CNN (RGB-D) & & 98 & & & & & & & & & & & & & 75 & \\
\hline 2018 & [88] & $\begin{array}{l}\mathrm{i} 3 \mathrm{D} C \mathrm{CNN}+2 \mathrm{DCNN} \\
\text { (RGB-D) }\end{array}$ & & & & & & & 92 & & & & & & & & 94 & \\
\hline 2019 & [95] & CNN (RGB + OF) & & & & & & & & 56 & & & & & & & & \\
\hline 2019 & [85] & i3D CNN (RGB) & & & & & & & & & & 74 & & & & & & \\
\hline 2019 & [96] & 3D CNN (RGB) & & & & & & & & & & & 75 & & & & & \\
\hline 2019 & [87] & GCN (Skeleton) & & & & & & & & & & & & 80 & & 91 & & \\
\hline 2019 & [97] & CNN (RGB) & & & & & & & & & & & & & 82 & & & \\
\hline 2019 & [98] & $\begin{array}{l}\text { TBN-Inception } \\
\text { Audio + OF) }\end{array}$ & & & & 35 & & & & & & & & & & & & \\
\hline 2020 & [99] & 3D CNN + GCN (RGB-D) & & & & & & 61 & & & 86 & & & & & & & \\
\hline 2020 & [100] & HAMLET (RGB-D) & & & 98 & & & & 95 & & & & & & & & & \\
\hline 2020 & [101] & CNN (RGB-D) & & & & & 94 & & 92 & & 95 & & & & & & 99 & \\
\hline
\end{tabular}

\section{Deep Learning}

Computer vision researchers have directed considerable attention to the application of deep learning in action recognition. The classical machine learning-based methods are based on handcrafted features, which are not robust. Deep learning-based methods have been utilized due to their automated feature learning from images. Researchers have extracted action features from RGB data, depth data, and skeleton sequences using deep learning methods. The following subsections discuss the fundamental variants of neural networks, and later we present some modern deep learning-based approaches used in RGB-D data.

\subsection{Neural Networks Variants}

Recent successes in deep neural networks have boosted research in pattern recognition and computer vision. Some commonly used variants of neural networks are briefly outlined in following subsections.

\subsubsection{Convolutional Neural Networks (CNN)}

CNNs represent one of the most notable deep learning approaches, where they have been highly effective in a variety of computer vision applications. CNNs are good at 
recognizing patterns in Euclidean data, i.e., images, text, and videos. CNN works with a mathematical function called convolution, which is a special kind of linear operation. In convolution, input neurons are multiplied with a set of weights that are commonly known as filters or kernels. The filters act as a sliding window across the whole image and enable CNNs to learn features from neighboring cells. Within the same layer, the same filter will be used throughout the image, this is referred to as weight sharing. For example, using a CNN to classify images of dogs vs. non-dogs, the same filter could be used in the same layer to detect the nose and the ears of the cat. There are four basic ideas behind CNN that benefit from the characteristics of natural signals: local connections, shared weights, pooling, and the use of many layers [70]. These four key ideas can be labeled as the Convolution layer, Rectified Linear Unit (ReLU) layer, Pooling, and Fully Connected (FC) Layer, respectively. An example of CNN architecture is presented in Figure 5a, originally shown in [71].

\subsubsection{Recurrent Neural Networks (RNN)}

The Recurrent Neural Network (RNN), Auto-Associative, or Feedback Network is a type of neural network that has variants including Gated Recurrent units. RNNs have been quite successful in conducting tasks like speech recognition, caption generation, machine translation, image/video classification, human dynamics, and action recognition, among other applications. The RNN function is an alternative to CNN; the RNN function is good at learning dependencies among spatially correlated data like image pixels [72]. RNN cannot store information for a longer duration. Long Short-Term Memory (LSTM) is a special kind of RNN capable of learning temporal relationships on a long-term scale. LSTM [73] uses a gates mechanism: write (input gate), read (output gate), and reset (forget gate), where this gates mechanism controls the behavior of its memory cells. The use of LSTM has produced effective results in speech recognition, especially in phoneme recognition. However, learning with LSTM is often challenging in real-time sequences [74]. An example of LSTM architecture is presented in Figure 5b, originally shown in [75].

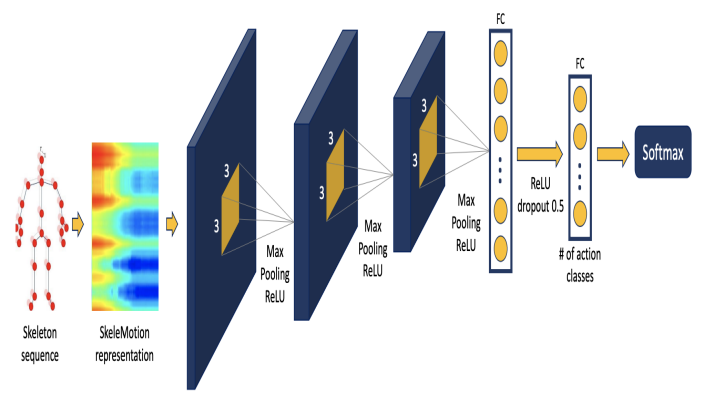

(a)

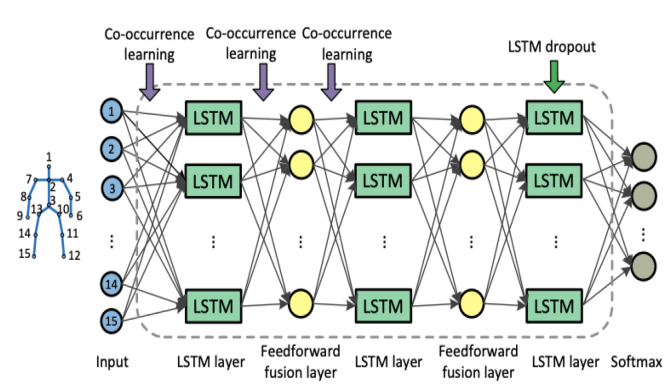

(b)

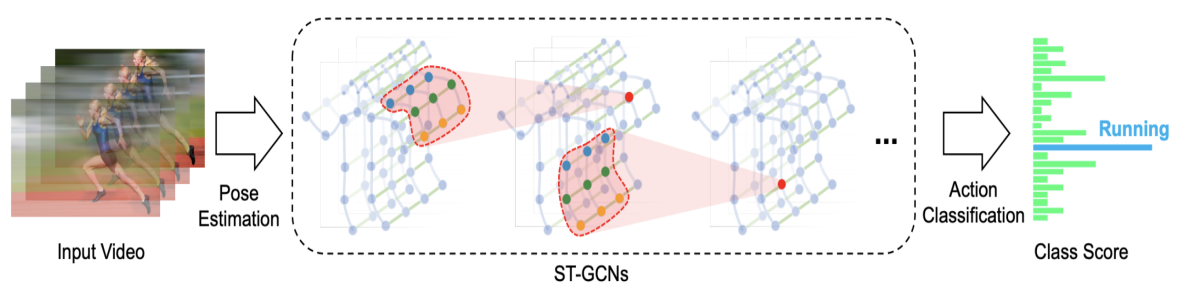

(c)

Figure 5. Illustration of deep learning techniques for processing RGB-D data. (a) Convolutional Neural Network (CNN). (b) Long Short-Term Memory (LSTM). (c) Graph Convolutional Network (GCN).

\subsubsection{Graph Convolutional Networks (GCN)}

Earlier variants of neural networks are implemented using regular or Euclidean data. However, real-life data have a graph structure that is non-Euclidean. Therefore, the non- 
regularity of data structures has led to advancements in graph neural networks. Graph Convolutional Networks (GCN) are also considered as one of the basic Graph Neural Networks variants. Convolution in GCNs is the same operation as in CNNs. GCNs [76] are an efficient variant of CNNs on graphs. In GCNs, the model learns from the neighboring nodes by stacking layers of learned first-order spectral filters followed by a nonlinear activation function to learn graph representations. For simplicity, GCNs take a graph with some labeled nodes as input and generate label predictions for all graph nodes. GCNs could be divided into two approaches: Spatial GCNs and Spectral GCNs. An example of GCN is presented in Figure 5c, originally shown in [77].

\subsection{Deep Learning-Based Techniques Using RGB-D Data}

Deep learning can directly obtain hierarchical features from different data modalities and provides a more effective solution. Accordingly, appearance and optical sequences can be used as inputs to deep networks. Besides aspects of appearance and motion information, deep learning-based methods can also be applied using depth sequences and skeleton joint information. Wang et al. [102] have used convolution to learn action features from depth data. They [103] combined motion and structure information in a depth sequence by pairing structured dynamic images at the body, part, and joint levels through bidirectional rank pooling. Every pair is constructed from depth maps at each granularity level and serves as input to CNN. Song et al. [104] have proposed a model that uses different levels of attention in addition to an RNN with LSTM to learn discriminative skeleton joints. Ye et al. [105] have embedded temporal information with dense motion trajectories to learn actions.

Yan et al. [71] have modeled relationships between graphs and joints by using a graph-oriented CNN. Deep learning-based feature learning has been shown to provide better performance than handcrafted feature extraction methods; however, there are still challenges concerning RGB-D data fusion. Deep learning-based action recognition methods use different standalone as well as hybrid neural network architectures, which can be classified as Single-Stream, Two-Stream, Long-term Recurrent Convolutional Network (LRCN), and Hybrid network-based architectures. The following subsections summarize these architectural styles.

\subsubsection{Single Stream}

A single-stream model is similar to the AlexNet [106] type of image classification network. Single-stream architecture can take advantage of regularization through local filters, parameter sharing at convolution layers, and local invariance building neurons (max pooling). Such neural network architecture shifts the engineering focus from feature design strategies to network structure and hyperparameter tuning strategies. Architectural details from AlexNet [106] can be used with different hyperparameter configurations. A single-stream architecture fuses information from all the frames in the softmax layer connected to the last fully connected layers with dense connections. Given an entire action video, the video-level prediction can be produced by forward propagation of each frame individually through the network and then averaging individual frame predictions over the duration of the video. However, single-stream architecture has been a foundation for other extended architectures. Some possible extensions to single-stream architecture have been explored by Baccouche et al. [107], Ji et al. [108], and Karpathy et al. [86].

\subsubsection{Two Stream}

The two-stream model uses two disjointed CNNs containing spatial and temporal information, which are later fused together. The spatial network performs action recognition from single video frames, while the temporal network learns to recognize action from motion, i.e., dense optical flow. The idea behind this two-stream model relates to the fact that the human visual cortex contains two pathways for object and motion recognition, i.e., the ventral stream performs object recognition and the dorsal stream recognizes motion. 
Spatial-stream CNN is modelled similar to the single-frame model discussed earlier. Given an action video, each frame is individually passed through the spatial network where an action label is assigned to each frame. The temporal-stream CNN is not the same as motion-aware CNN models (which use stacked single video frames as input). It takes stacked optical flow displacement fields between several consecutive frames as input to explicitly learn a temporal feature.

In two-stream models, the pioneering work of Simonyan and Zisserman [109] uses a single image and multi-optical flow sequence stack as input to the 2D CNN. Zhang et al. [110] have extended Simonyan and Zisserman's [109] work by using a motion vector instead of optical flow as an input to improve performance and comprehend real-time action recognition. Feichtenhoer et al. [111] have proposed an innovative approach involving moving the classification layer to the middle of the network for spatio-temporal information fusion, and this was shown to improve the accuracy. Wang et al. [112] have contributed to the input and training strategy of convolution and proposed Temporal Segment Network (TSN), improving the two-stream CNN. The notion of TSN was based on long-range temporal structural modeling. Later, Lan [113] and Zhou [114] enhanced the TSN. Carreira et al. [115] adopted the structure of Inception-v1 and inflated two-stream $\mathrm{CNN}$ to 3D CNN for action recognition. Zhu et al. [116] have expanded two-stream CNN to a 3D structure by drawing out the pooling operation.

\subsubsection{Long-Term Recurrent Convolutional Network (LRCN)}

LRCN uses CNN in co-ordination with an LSTM-based network. In LSTM-based deep learning methods, actions can be represented as feature changes between frames in the video, LSTM is widely used to improve action recognition techniques. Ng et al. [117] have presented a linear RNN for recognizing human actions that connects the output of a CNN with an LSTM cell. A new architecture-P3D ResNet-has been proposed by Qiu et al. [118], which uniquely places all the variants of blocks in a different placement of ResNet. In skeletal data, to deal with noise, Liu et al. [119] extended the idea to analyze spatio-temporal domains simultaneously by introducing an effective tree structure-based traversal framework. This framework uses a cross-modal feature fusion strategy within LSTM unit and a gating mechanism to learn the reliability of sequential data in long-term context representation. For mapping video frames with variable length inputs to variable length outputs, Donahue et al. [120] have proposed an LRCN. Unlike those methods that learn CNN filters based on a stack of a fixed number of input frames, LRCN [120] is not constrained to fixed-length input frames and thus can learn to recognize more complex action video. As illustrated in Figure 6 (RGB-D input visuals taken from the work in [31]), individual video frames are first passed through $\mathrm{CNN}$ models with shared parameters and are then connected to a single-layer LSTM network. More precisely, the LRCN model combines a deep hierarchical visual-feature extractor, i.e., a CNN feature extractor, with an LSTM that can learn to recognize temporal variations in an end-to-end fashion.

\subsubsection{Hybrid Deep Learning-Based Techniques for HAR}

Hybrid deep learning-based approaches that use advanced variants of deep learning architectures to produce state-of-the-art action recognition accuracy on popular datasets are discussed below.

On a large-scale YT-8M dataset, Abu-El-Haija et al. [121] pretrained an LSTM network and discovered that pretraining on a large-scale data set generalizes well on datasets such as Sports-1M [86] and ActivityNet [122]. However, Jinyoung et al. [123] proposed an extensible hierarchical method for detecting generic interactive actions (by combining spatial relations with movements between two objects) and inherited actions determined through an ontology of rule-based methodology. This technique outperforms other techniques on the ActionNet-VE dataset [124]. 


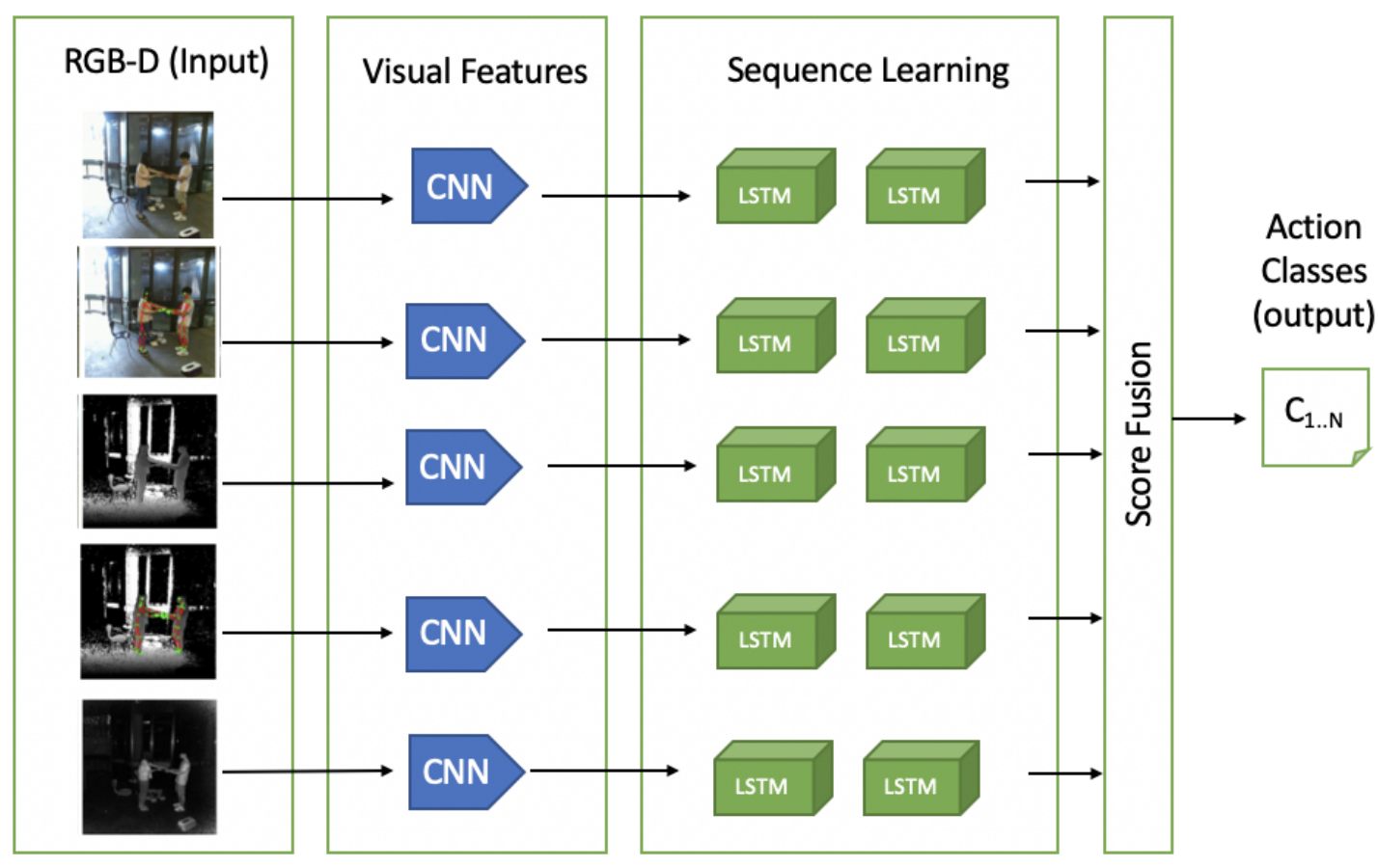

Figure 6. A possible architecture of LRCN with RGB-D input. Input from each modality, i.e., RGB, RGB + Skeleton joints, Depth, Depth + Skeleton joints, and IR are passed through a CNN layer for extracting visual features and an LSTM layer for sequence learning. Scores from each model are then fused and mapped to the number of classes for predictions. Visuals of RGB-D input are taken from NTU RGB-D 60 dataset [90].

Yuan et al. [125] have proposed the Multi-Granularity Generator (MGG), which produces temporal action proposals through two producers: the Segment Proposal Producer (SPP) and the Frame Actionness Producer (FAP). SPP generates a segment proposal from a coarse perspective, while FAP produces a finer actionness evaluation for each video frame. Both producers are combined to reflect two different granularities. MGG can be trained in an end-to-end fashion and performs better than state-of-the-art methods on the THUMOS-14 dataset. In addition, Zhaofan et al. [126] have provided a novel neural network architecture that uses spatio-temporal representation learning by Local and Global Diffusion (LGD) in parallel. This architecture is composed of LGD blocks, which update local and holistic features. In addition to this, a kernelized classifier is used for video recognition.

An efficient and generic Temporal Shift Module (TSM) was proposed by Lin et al. [127] which claims the performance of 3D CNN while maintaining the complexity of 2D CNN. TSM facilitates information sharing among neighboring frames by shifting a portion of the channels along the temporal dimension. TSM achieves state-of-the-art results on the Something-Something dataset. However, Zhu et al. [88] have initiated the idea of an Action Machine which is a simple fast method extended from an Inflated 3D CNN by adding a module of 2D CNN and pose estimation. The Action Machine takes input that is cropped through person-bounding boxes, fusing predictions from RGB images and poses. Action Machine produced state-of-the-art results on NTU RGB-D datasets and competitive results on smaller datasets of Northwestern-UCLA, MSR-DailyActivity3D, and UTD-MHAD [83,91].

Girdhar et al. [128] have proposed the Video Action Transformer Network (VATN) which uses transformer-style architecture that aggregates features from the spatio-temporal context. VATN uses an attention mechanism that learns to emphasize hands and faces. On the Atomic Visual Actions (AVA) dataset, VATN outperformed state-of-the-art methods by using raw RGB frames as input. Moreover, Hu et al. [129] have explored the modalitytemporal mutual information to learn time-varying information and cross-modal features 
jointly. They introduced an action feature called a modality-temporal cube, which characterizes RGB-D actions from a comprehensive perspective. Their proposed framework uses deep bilinear blocks that pool input from both modality and temporal directions.

On the other hand, the concept of the Gate Shift Module (GSM) in the spatio-temporal decomposition of 3D kernels was introduced by Sudhakaran et al. [130]. GSM is added to a 2D CNN that learns route features and combines them with less computational complexity and additional parameters overhead. This technique achieves state-of-the-art results on the Something-Something-v1 dataset and competitive results on other datasets. In addition, Caetano et al. [77] have proposed the Skelemotion, which extends Spatio-Temporal Graph Convolutional Networks (ST-GCN) by introducing a Graph Vertex Feature Encoder (GVFE) and Dilated Hierarchical Temporal Convolutional Network (DH-TCN). GVFE learns vertex features by encoding raw skeleton features data, while DH-TCN captures both shortand long-term dependencies. This architecture uses fewer layers and parameters, and it competes better with state-of-the-art methods on NTU RGB-D 60 and NTU RGB-D 120 datasets.

Korbar et al. [85] have introduced a clip sampling scheme that selects salient temporal clips within a long video. This technique improves the state-of-the-art and reduces computational costs significantly. However, Wang et al. [96] have proposed Channel-Separated Convolutional Networks (CSNN) which demonstrate the benefits of factorizing 3D convolutions by separating spatio-temporal interactions and channel interactions. The latter is a form of regularization that improves accuracy and lowers computational costs.

In [87], Object-Related Human Action recognition through Graph Convolution Networks (OHA-GCN) was proposed which constructs graphs using selective sampling of human and object poses. OHA-GCN late fuses class scores from human poses and object pose streams for action classification. Furthermore, Wang et al. [97] have proposed a network that creates improved Dense Trajectories (iDT) descriptors and i3D optical flow features with $\mathrm{CNNs}$, thus reviving classical handcrafted representations.

Liu et al. [131] have introduced a novel neural network called CPNet that learns evolving 2D fields with temporal consistency. CPNet achieved state-of-the-art results on both Jester [132] and Something-Something datasets [127,130]. Moreover, Martin et al. [133] have introduced the novel approach for fine-grained categorization of driver behavior. They focused on key challenges such as recognition of fine-grained behavior inside the vehicle cabin, focusing on diverse data streams and a cross-view recognition benchmark and adopting prominent methods for video and body pose-based action recognition to provide challenging benchmarks. Besides, Munro and Damen [134] have exploited the correspondence of modalities as a self-supervised alignment approach in addition to adversarial alignment, which outperforms other unsupervised domain adaptation methods.

Table 3 summarizes the key differences among deep learning-based action recognition methods that have evolved over the last decade. It can be observed that most of the techniques are not applied on RGB-D datasets. Accuracy is the most preferred metric for action recognition, where sometimes these techniques benefit from the use of extra training data. 
Table 3. State-of-the-art action recognition techniques with their key differences. Notations: CA: Code Availability; ET: Extra Training; TL: Transfer Learning; Y: Yes; N: No; mAP: Mean Average Precision; OF: Optical Flow; IR: Infrared; FV: Fisher Vectors; BoW: Bag of Words; iDT: improved Dense Trajectories; E:Early, M:Middle; L:Late.

\begin{tabular}{|c|c|c|c|c|c|c|c|c|}
\hline Ref. & $\mathrm{CA}$ & ET & TL & Metric & Network/Classifier & Modality & Fusion & Novelty \\
\hline [92] & $\mathrm{N}$ & $\mathrm{N}$ & $\mathrm{N}$ & Accuracy & SVM & RGB-D & $\mathrm{M}$ & $\begin{array}{l}\text { Extracts interest points solely from } \\
\text { RGB channels and combines RGB } \\
\text { and depth map-based desciptors. }\end{array}$ \\
\hline [93] & $\mathrm{N}$ & $\mathrm{N}$ & $\mathrm{N}$ & Accuracy & SVM & RGB-D & $\mathrm{L}$ & $\begin{array}{l}\text { Modelling of temporal dynamics of } \\
\text { human actions by temporal order } \\
\text { preserving dynamic quantization } \\
\text { method. }\end{array}$ \\
\hline [94] & $\mathrm{N}$ & $\mathrm{N}$ & $\mathrm{N}$ & Accuracy & $\mathrm{CNN}+\mathrm{SVM}$ & RGB-D & M & $\begin{array}{l}\text { Deep hierarchical shared-specific } \\
\text { defactorization of RGB-D features } \\
\text { and a structured sparsity learning } \\
\text { machine. }\end{array}$ \\
\hline [96] & $\mathrm{N}$ & $\mathrm{N}$ & $\mathrm{Y}$ & Accuracy & 3D CNN & RGB & - & $\begin{array}{l}\text { Separated spatio-temporal } \\
\text { interactions. }\end{array}$ \\
\hline$[88]$ & $\mathrm{Y}$ & $\mathrm{Y}$ & $\mathrm{Y}$ & Accuracy & i3D CNN & RGB + Pose & $\mathrm{L}$ & $\begin{array}{l}\text { Used person cropped frames as } \\
\text { inputs. }\end{array}$ \\
\hline [95] & $\mathrm{N}$ & $\mathrm{Y}$ & $\mathrm{Y}$ & $\mathrm{mAP}$ & $\mathrm{CNN}$ & $\mathrm{RGB}+\mathrm{OF}$ & $\mathrm{L}$ & $\begin{array}{l}\text { Reformulated neural architecture } \\
\text { search for video representation. }\end{array}$ \\
\hline [85] & $\mathrm{N}$ & - & $\mathrm{Y}$ & Accuracy & i3D CNN & $\begin{array}{l}\mathrm{IR}+\mathrm{OF}+ \\
\mathrm{RGB}\end{array}$ & M & $\begin{array}{l}\text { Used salient clip sampling to } \\
\text { improve efficiency. }\end{array}$ \\
\hline [77] & $\mathrm{Y}$ & $\mathrm{N}$ & $\mathrm{N}$ & Accuracy & $\mathrm{CNN}$ & Skeleton & $\mathrm{E}$ & $\begin{array}{l}\text { Employed graph vertex encoding } \\
\text { along with few layers and } \\
\text { parameters. }\end{array}$ \\
\hline$[87]$ & $\mathrm{N}$ & $\mathrm{N}$ & $\mathrm{N}$ & Accuracy & GCN & Skeleton & - & Used human-object related poses. \\
\hline [97] & $\mathrm{N}$ & $\mathrm{Y}$ & $\mathrm{N}$ & Accuracy & $\mathrm{CNN}$ & $\mathrm{iDT} / \mathrm{FV} / \mathrm{BoW}$ & M & $\begin{array}{l}\text { Combined classical handcrafted } \\
\text { iDT features with CNN extracted } \\
\text { features. }\end{array}$ \\
\hline$[98]$ & Y & $\mathrm{N}$ & $\mathrm{Y}$ & Accuracy & 3D-CNN + GCN & $\begin{array}{l}\text { RGB-D + OF } \\
\quad+\text { Audio }\end{array}$ & M & $\begin{array}{l}\text { Architecture for multimodal } \\
\text { temporal binding. }\end{array}$ \\
\hline [99] & Y & $\mathrm{Y}$ & Y & Accuracy & $3 \mathrm{D}-\mathrm{CNN}+\mathrm{GCN}$ & RGB-D & M & $\begin{array}{l}\text { A spatial embedding with an } \\
\text { attention network. }\end{array}$ \\
\hline [100] & $\mathrm{N}$ & $\mathrm{N}$ & $\mathrm{Y}$ & Accuracy & CNN & RGB-D & M & $\begin{array}{l}\text { Multimodal attention mechanism } \\
\text { for disentangling and fusing the } \\
\text { salient features. }\end{array}$ \\
\hline [101] & $\mathrm{N}$ & $\mathrm{N}$ & $\mathrm{Y}$ & Accuracy & $\mathrm{CNN}$ & Skeleton & - & $\begin{array}{l}\text { Inflated ResNet coupled with } \\
\text { hierarchical classification and } \\
\text { iterative pruning. }\end{array}$ \\
\hline
\end{tabular}

In HAR, the implementation and execution of deep learning-based methods can often be time-consuming. Experimental platforms provide abstraction, customization, community, and advanced hardware-level support. This is important for the development of robust and flexible deep learning-based action recognition systems. Some platforms are intuitive and highly abstract, but such abstractions or wrappers can make it difficult to debug or apply explicit changes to algorithms at low levels. As performance demand relies on high-end hardware and multiple graphical processing units (GPU), support is a must when experimenting with big data-related problems. 


\section{Data Fusion Techniques}

Data fusion supports diversity, which enhances the uses, advantages, and analysis of ways that cannot be achieved through a single modality. Fusion techniques can be deployed at different stages in the action recognition process to acquire combinations of distinct information. The following are some popular ways of fusing action data in RGB-D datasets.

\subsection{Early Fusion}

The early fusion approach captures information and combines it across a raw or data level. To achieve this, the filters on the first layer in the neural network are modified. This direct and early connectivity of the raw data helps the network to detect the fused feature vectors at an early stage. For an entire action data sequence, some randomly selected sample instances are often passed through the system, and their class predictions are then averaged to produce action class predictions, as illustrated in Figure 7a (adapted from the work in [135]).

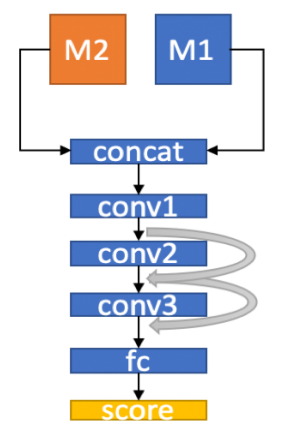

(a)

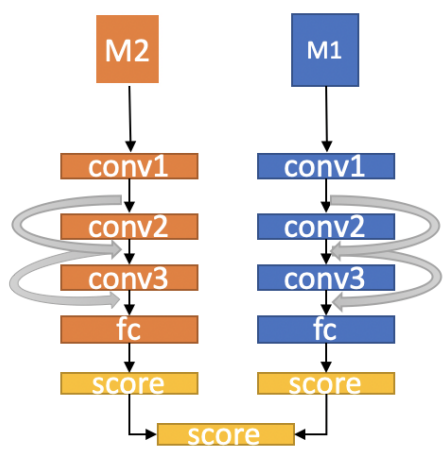

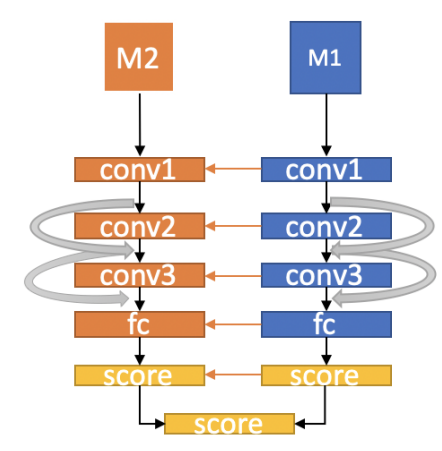

(b)

(c)

Figure 7. An example of (a) early, (b) slow, and (c) late fusion in HAR. Note that the input modalities are not limited in the above two modalities.

\subsection{Slow Fusion}

The slow fusion approach fuses the features extracted from raw data throughout the neural network so that the higher layers have access to more global information. This is achieved by performing a CNN-, RNN-, or LSTM-based operation to calculate the weights and extend the connectivity of all layers. For example, as shown in Figure $7 \mathrm{~b}$, raw data from two-different modalities are filtered in the first convolution layer. The next layers iterate this process in the network with different filter configurations. Therefore, the information across all the input data can be assessed by the subsequent convolution layers. Given an entire human action data sequence, action classification is performed over the entire dataset through the network and then averaging individual predictions throughout the action sequence. 


\subsection{Late Fusion}

The late fusion approach combines the action information at the deepest layers in the network, for example, a HAR architecture network consisting of two separate CNN-based networks with shared parameters up to the last convolution layer. The outputs of the last convolution layer of these two separate network streams are processed to the fully connected layer. Global action characteristics are fused, and the classification score is then averaged or concatenated by different holistic operations at the score layer. This approach has been relatively successful in most of the HAR systems. An illustration of late fusion is shown in Figure 7c.

\subsection{Multi-Resolution}

In order to speed up the above-mentioned models while retaining their accuracy, a multi-resolution architecture has been proposed by Karpathy et al. [86]. The multiresolution model consists of two separate networks (fovea and cortex networks) over two spatial resolutions. The architectures of fovea and cortex networks are similar to the singleframe architecture. However, instead of accepting the original input, these networks record reduced sized inputs. More precisely, the input to the fovea model is the center region at the original spatial resolution. In contrast, for the context stream, the downsampled frames at half the original resolution are used. The total dimensionality of the inputs is, therefore, halved. Moreover, the last pooling layer is removed from both the fovea and cortex networks. The activation outputs of both networks are concatenated and fed into the first fully connected layer.

All the above-mentioned network variations were trained on the Sports-1M dataset [86], which consists of 200,000 test videos. The results showed that the variation among different CNN architectures (e.g., Single Frame, Multi-Resolution, Early, Late, and Slow Fusion) was surprisingly insignificant. Furthermore, the results were significantly worse than the state-of-the-art handcrafted shallow models. This may be because these models cannot capture the motion information in many cases. For example, the slow fusion model is expected to implicitly learn the spatio-temporal features in its first layers, which is a difficult task. To resolve this issue, a two-stream CNN model was proposed by Simnonyan and Zisserman [109] to explicitly take into account both spatial and temporal information in a single end-to-end learning framework.

\section{Applications of State-of-the-Art RGB-D Methods}

RGB-D based Human action recognition (HAR) is a widely studied computer vision problem. As the imaging technique advances and the camera device upgrades, novel approaches for HAR constantly emerge. Some significant application areas are discussed below.

\subsection{Content-Based Video Summarization}

At present, the enormous use of multimedia devices has given growth to video content. The manual task of video content retrieval is time-consuming and tedious. The authors of [136] used color and texture features to demonstrate the benefit of different feature combinations for video summarization. The work in [137] proposed a real-time video summary by using a threshold based on the probability distribution. Identical features were removed by using redundancy elimination techniques.

\subsection{Education and Learning}

Classifying human actions from RGB-D data plays an important role in education and learning. The exploration of human actions based on visual data in educational institutions can help in recognition and predetermined monitoring of attendance during class. During this process, the instructor-led attendance is time-consuming and requires rigorous observation from the instructor. 
With the recent advances in technology, the automated attendance system can now be deployed in a classroom environment. To monitor student attendance, visual data are acquired to register students when they enter or leave the classroom. In [138], the system recognizes students and their activities such as entering and leaving the classroom. The system performs student identification by performing face recognition and motion analysis and performs action recognition to recognize students' actions. A combination of techniques using RGB-D data can be applied in different education and learning environments for efficient action recognition systems.

\subsection{Healthcare Systems}

The use of action recognition techniques using RGB-Data can benefit healthcare systems. For example, as the elderly are susceptible to disease, healthcare for the elderly has been a major concern. Automated monitoring systems are required to recognize actions such as falls and other abnormal behaviors in the elderly. In [139], the authors proposed an approach to depict the behavior of dementia (Alzheimer's and Parkinson's) in patients. RNN variants such as Vanilla RNNS, LSTM, and Gated Recurrent Unit (GRU) are used to detect abnormal actions in elderly patients with dementia. Continuous monitoring of temperature, blood pressure, blood glucose, and blood oxygen is performed using different smartphone sensors. A warning is generated by the system in case of abnormal activity in [140].

\subsection{Entertainment Systems}

HAR has been broadly explored to recognize actions in dance moves. Laptev et al. [141] presented the task of recognizing actions using text-based classifier (regulated perceptron). Space-time functions and nonlinear SVMs are used to classify actions from the film script. In addition, Wang et al. [142] classified film actions using 3D CNN. Two modules, namely, coding and a temporal pyramid pooling layer, were used introduced in order to minimize the loss during learning. A feature linkage layer was incorporated to combine motion and appearance information. HAR is also used to detect dance moves using videos. Kumar et al. [143] analyzed the dataset based on classical Indian dance using an AdaBoost classifier with multiple classes and merged characteristics. On the other hand, Castro et al. [144] discovered that visual information in motion-intensive videos is insufficient to efficiently recognize actions. The experimentation is performed using RGB, optical flow and multi-person pose data.

\subsection{Safety and Surveillance Systems}

RGB-D-based HAR techniques can be used to ensure safety in public venues such as railway stations and airports. Action recognition in such an environment is challenging due to a large number of viewpoints. An abnormal activity can be detected even for objects following the same pattern. For example, a train crossing the railway line is considered a normal activity, while a person crossing a railway line is considered as an abnormal activity. In [145], the authors presented an efficient intelligent system for crowded scene using a deep Gaussian mixture model. The multi-layer nonlinear input transformation improves the performance of the network with a few parameters. HAR can also be used to identify abnormal activity recognition in (Unmanned Aerial Vehicle) UAV-based surveillance [146].

\subsection{Sports}

Highlighting key moments in sports videos is difficult for coaches and players to analyze; it can be time-consuming and uninteresting for the audience to watch long games continuously [147]. Recent studies focus on analyzing player movement individually and in groups for their training and evaluation. Ullah et al. [148] used the pretrained deep CNN model VGG-16 to identify player actions. A deep autoencoder is used to learn changes over time and human actions are classified using SVM. In group activities, graph-based are 
widely used. Qi et al. [149] classified sports videos using a semantic diagram and an RNN is used to extend the semantic graph model to the temporal dimension.

\section{Challenges and Future Research Directions}

Action recognition remains challenging due to background clutter, partial occlusion, viewpoint, lighting changes, execution rate, and biometric variation. Challenges with data fusion followed by future research directions are discussed below.

\subsection{Challenges in RGB-D Data Fusion}

Deep learning-based techniques in HAR use various data fusion approaches such as early, slow, late fusion, and other variants. Some prominent challenges with data fusion approaches are discussed below.

- $\quad$ RGB-D datasets with different resolutions possesses an inherent challenge in data fusion because each modality has a very different temporal and spatial resolution.

- Practically, individual datasets contain incompatible numbers of data samples, which leads to data size incompatibility. Alignment of modalities to a standard coordinate system for maximizing mutual information sharing is an acute challenge in data fusion.

- Inherently, the information conveyed through each modality has different physical properties, which can be vital for better action learning. Identification of key characteristics from each modality that is contributing towards the overall recognition is an interesting problem.

- Negligible errors produced by RGB-D sensors are often abstracted as noise, which is unavoidable. Balancing noise with other modalities also causes problems in data fusion.

- Most data fusion techniques ignore the noise, but ignoring the noise from datasets collected through different sensors may lead to bias.

- Distinct data modalities confront contradictions, and data inconsistencies may occur. An open challenge is to infer a proper compromise; however, identifying these conflicts, contradictions, and inconsistencies is a fundamental challenge.

- $\quad$ RGB-D sensors may produce spurious data due to environmental or sensor failure issues, which may lead to false inferences based on biased estimations. Therefore, a challenge may arise in predicting and modeling spurious events.

- Other challenging factors include noise, spatial distortions, varying contrast, and arbitrary subject locations in image sequences.

Data fusion techniques vary across different tasks and need to address various challenges in terms of required time and memory management. Other problems may arise depending on the modality under consideration. For example, basic skeleton features (joint coordinates or bone lengths) are used commonly for constructing spatio-temporal graphs. However, offering a high-level description of the human body structure may affect discriminative power for action recognition. However, an innovative strategy for the combining or augmenting of different modalities at an earlier or any later phase of resolution can lead to better data fusion solutions.

\subsection{Future Research Directions}

The discussion and insights drawn from the challenges in different approaches allow us to present several future research directions to develop methods in action recognition. The following research directions may advance the domain.

\subsubsection{Combination of Classical Machine Learning and Deep Learning-Based Methods}

Classical machine learning approaches have benefited action recognition through redundant and favorable feature extraction. Deep learning-based methods provide autonomous feature engineering and have produced better recognition systems. Designing effective action recognition systems by adding the power of classical machine learning 
with advanced deep learning-based techniques has some attraction for researchers. For example, Gao et al. [150] proposed a fusion logic of classical machine learning and deep learning-based methods to achieve better performance than single CNN-based pedestrian detector, and it is likely to emerge as an active research area.

\subsubsection{Assessment in Practical Scenarios}

Most of the RGB-D datasets have been collected in constraint environments. There remains a significant gap between the collected datasets during the last few years and the practical scenario due to insufficient categories, occlusion cases, constrained environment settings, samples, and limited distance variations. Due to these limitations, collected datasets may not substitute the need for outdoor practical scenario-based datasets. Collection and generalization of algorithms over realistic scenarios should gain the attention of researchers.

\subsubsection{Self-Learning}

Learning labels about individual samples is often overlapping and causes inefficient intra-class similarity. Self-learning action recognition systems can learn from non-labeled training data without any human intervention. Recent advances in deep learning, such as Generative Adversarial Networks (GAN), may improve action recognition systems' self-learning capability. GAN-based action recognition techniques would be a compelling research direction.

\subsubsection{Interpretation of Online Human Actions}

Action recognition algorithms focus on well-trimmed segmented data splits. While in an online action recognition system, which aims to observe many mechanisms such as facial expression, visual focus, view angles etc. instantly from a video stream. Interpretation of such human behavior components in online scenarios is an essential step toward more practical and intelligent recognition systems.

\subsubsection{Multimodal Fusion}

Multimodal data provide richer information than unimodal data. Still, most methods fuse different modalities as separate channels and combine them at a later classification stage without exploiting their corresponding properties in a parallel fashion. Effective use of deep networks for parallel integration of complementary properties from different modalities would a potential research area. Use of multimodal information also helpful in reducing noise from unimodal data. Therefore, integrating multimodal information and incorporating contextual information from the surrounding environment is a way forward for future research. Different fusion schemes are used in various methods for action classification. Thus, future research may devote more attention from researchers to compare these fusion schemes and find the best fusion strategy for action recognition.

\section{Conclusions}

The vision-based recognition of human actions is an important research field in the integrative computer vision and multimedia analytics ecosystem. This review has thoroughly compared and summarized the landscape of vision-based RGB-D sensors. We provided an outline of existing commonly used datasets and highlighted key research that has mainly focused on RGB-D datasets. We also reviewed the latest action recognition techniques that use deep learning in general. We discussed the techniques that have been used over the past decade and divided them into different perspectives. We then presented various available experimental options, along with their characteristics, strengths, and weaknesses, for action recognition researchers.

The results of this paper show that with the availability of low-cost and multi-function sensors, the effects of RGB-D action recognition can be extended to wider application areas. It is evident that deep learning architectures, especially CNN- and LSTM-based 
methods, have been shown to produce significant results. However, there is a lack of availability of large data sets in different domains. Attention has turned more to RGB, optical flow, and skeletal modalities, so other promising modalities such as depth and IR have not been adequately explored. The challenges are evident with RGB-D action sensors, data sets, recognition, and fusion techniques. Significant efforts are required to address these challenges.

Author Contributions: Conceptualization, M.B.S. and D.C.; methodology, M.B.S.; software, M.B.S.; validation, D.C.; formal analysis, M.B.S.; investigation, M.B.S.; data curation, M.B.S.; writing—original draft preparation, M.B.S.; writing—-review and editing, D.C.; visualization, M.B.S.; supervision, D.C.; project administration, D.C.; funding acquisition, D.C. All authors have read and agreed to the published version of the manuscript.

Funding: This research was funded by a joint research project grant (No.5-1/HRD/UESTPI(BatchVI) $/ 7108 / 2018 /$ HEC) of the Higher Education Commission (HEC) Pakistan and Edith Cowan University (ECU) Australia.

Institutional Review Board Statement: Ethical review and approval were waived for this study, as it does not involve any humans or animals. Edith Cowan University Research Ethics Reference No. 2019-00764-SHAIKH.

Informed Consent Statement: Informed consent was obtained from all subjects involved in the study.

Data Availability Statement: Not Applicable.

Acknowledgments: The authors would like to thank the anonymous reviewers for their careful reading and valuable remarks, which have greatly helped extend the scope of this paper. All statements of fact, opinion, or conclusions contained herein are those of the authors and should not be construed as representing the official views or policies of the sponsors.

Conflicts of Interest: The authors declare no conflicts of interest. The funders had no role in the design of the study; in the collection, analyses, or interpretation of data; in the writing of the manuscript; or in the decision to publish the results.

\section{Abbreviations}

The following abbreviations are used in this manuscript:

$\begin{array}{ll}\text { AME } & \text { Accumulation of Motion Energy } \\ \text { AVA } & \text { Atomic Visual Actions } \\ \text { CSNN } & \text { Channel Separated Convolutional Networks } \\ \text { CNN } & \text { Convolutional Neural Network } \\ \text { DH-TCN } & \text { Dilated Hierarchical Temporal Convolutional Network } \\ \text { FAP } & \text { Frame Actionness Producer } \\ \text { GPU } & \text { Graphics Processing Unit } \\ \text { GVFE } & \text { Graph Vertex Feature Encoder } \\ \text { HoD } & \text { Histograms of Oriented Displacements } \\ \text { iDT } & \text { improved Dense Trajectories } \\ \text { IR } & \text { Infrared } \\ \text { HoG } & \text { Histogram of Gradients } \\ \text { LGD } & \text { Local and Global Diffusion } \\ \text { LRCN } & \text { Long-term Recurrent Convolutional Network } \\ \text { LSTM } & \text { Long-Short Term Memory } \\ \text { mAP } & \text { mean Average Precision } \\ \text { MGG } & \text { Multi-Granularity Generator } \\ \text { OHA-GCN } & \text { Object-Related Human Action recognition through Graph Convolution Networks } \\ \text { RGB } & \text { Red Green Blue } \\ \text { RGB-D } & \text { Red Green Blue-Depth } \\ \text { RNN } & \text { Recurrent Neural Network }\end{array}$


SPP Segment Proposal Producer

ST-GCN Spatio-Temporal Graph Convolutional Networks

SVM Scalar Vector Machines

TSM Temporal Shift Module

VATN Video Action Transformer Network

\section{References}

1. Yang, L.; Zhang, L.; Dong, H.; Alelaiwi, A.; Saddik, A.E. Evaluating and Improving the Depth Accuracy of Kinect for Windows v2. IEEE Sens. 2015, 15, 4275-4285. [CrossRef]

2. Carfagni, M.; Furferi, R.; Governi, L.; Santarelli, C.; Servi, M.; Uccheddu, F.; Volpe, Y. Metrological and Critical Characterization of the Intel D415 Stereo Depth Camera. Sensors 2019, 19, 489. [CrossRef]

3. Yeung, L.F.; Yang, Z.; Cheng, K.C.C.; Du, D.; Tong, R.K.Y. Effects of camera viewing angles on tracking kinematic gait patterns using Azure Kinect, Kinect v2 and Orbbec Astra Pro v2. Gait Posture 2021, 87, 19-26. [CrossRef]

4. Herath, S.; Harandi, M.; Porikli, F. Going Deeper into Action Recognition: A Survey. Image Vis. Comput. 2017, 60, 4-21. [CrossRef]

5. Aggarwal, J.; Cai, Q. Human Motion Analysis: A Review. Comput. Vis. Image Underst. 1999, 73, 428-440. [CrossRef]

6. Guo, G.; Lai, A. A Survey on Still-Image-based Human Action Recognition. Pattern Recognit. 2014, 47, 3343-3361. [CrossRef]

7. Poppe, R. A Survey on Vision-based Human Action Recognition. Image Vis. Comput. 2010, 28, 976-990. [CrossRef]

8. Turaga, P.; Chellappa, R.; Subrahmanian, V.S.; Udrea, O. Machine Recognition of Human Activities: A Survey. IEEE Trans. Circuits Syst. Video Technol. 2008, 18, 1473-1488. [CrossRef]

9. Wang, H.; Kläser, A.; Schmid, C.; Cheng-Lin, L. Action Recognition by Dense Trajectories. In Proceedings of the IEEE Computer Society Conference on Computer Vision and Pattern Recognition (CVPR), Providence, RI, USA, 16-20 June 2011; pp. $3169-3176$.

10. Zhu, G.; Zhang, L.; Mei, L.; Shao, J.; Song, J.; Shen, P. Large-scale Isolated Gesture Recognition using Pyramidal 3D Convolutional Networks. In Proceedings of the 23rd International Conference on Pattern Recognition, Cancun, Mexico, 4-8 December 2016; pp. 19-24.

11. Asadi-Aghbolaghi, M.; Clapés, A.; Bellantonio, M.; Escalante, H.J.; Ponce-López, V.; Baró, X.; Guyon, I.; Kasaei, S.; Escalera, S. A Survey on Deep Learning Based Approaches for Action and Gesture Recognition in Image Sequences. In Proceedings of the International Conference on Automatic Face Gesture Recognition, Washington, WA, USA, 30 May-3 June 2017; pp. 476-483.

12. Prince, S. Computer Vision: Models, Learning, and Inference, 1st ed.; Cambridge University Press: Cambridge, MA, USA, 2012.

13. Szeliski, R. Computer Vision: Algorithms and Applications, 1st ed.; Springer: London, UK, 2010.

14. Wang, P.; Li, W.; Ogunbona, P.; Wan, J.; Escalera, S. RGB-D-based Human Motion Recognition with Deep Learning: A Survey. Comput. Vis. Image Underst. 2018, 171, 118-139. [CrossRef]

15. Aggarwal, J.; Xia, L. Human Activity Recognition from 3D Data: A Review. Pattern Recognit. Lett. 2014, 48, 70-80. [CrossRef]

16. Chen, L.; Wei, H.; Ferryman, J. A Survey of Human Motion Analysis using Depth Imagery. Pattern Recognit. Lett. 2013, 34, 1995-2006. [CrossRef]

17. Han, F.; Reily, B.; Hoff, W.; Zhang, H. Space-time Representation of People based on 3D Skeletal Data: A Review. J. Vis. Commun. Image Represent. 2017, 158, 85-105. [CrossRef]

18. Zhang, J.; Li, W.; Ogunbona, P.O.; Wang, P.; Tang, C. RGB-D-based Action Recognition Datasets: A Survey. Pattern Recognit. 2016, 60, 86-105. [CrossRef]

19. Ye, M.; Zhang, Q.; Wang, L.; Zhu, J.; Yang, R.; Gall, J. A Survey on Human Motion Analysis from Depth Data. In Time-of-Flight and Depth Imaging. Sensors, Algorithms, and Applications, Lecture Notes in Computer Science; Springer: Berlin, Germany, 2013; Volume 8200, pp. 149-187.

20. Zhu, F.; Shao, L.; Xie, J.; Fang, Y. From Handcrafted to Learned Representations for Human Action Recognition: A Survey. Image Vis. Comput. 2016, 55, 42-52. [CrossRef]

21. Zhang, H.B.; Zhang, Y.X.; Zhong, B.; Lei, Q.; Yang, L.; Du, J.X.; Chen, D.S. A Comprehensive Survey of Vision-Based Human Action Recognition Methods. Sensors 2019, 19, 1005. [CrossRef]

22. Chen, C.; Jafari, R.; Kehtarnavaz, N. A Survey of Depth and Inertial Sensor Fusion for Human Action Recognition. Multimed. Tools Appl. 2017, 76, 4405-4425. [CrossRef]

23. Zhang, Z.; Ma, X.; Song, R.; Rong, X.; Tian, X.; Tian, G.; Li, Y. Deep Learning-based Human Action Recognition: A Survey. In Proceedings of the Chinese Automation Congress (CAC), Jinan, China, 20-22 October 2017; pp. 3780-3785.

24. Minh Dang, L.; Min, K.; Wang, H.; Jalil Piran, M.; Hee Lee, C.; Moon, H. Sensor-based and Vision-based Human Activity Recognition: A Comprehensive Survey. Pattern Recognit. 2020, 108, 107561. [CrossRef]

25. Sun, Z.; Liu, J.; Ke, Q.; Rahmani, H.; Bennamoun, M.; Wang, G. Human Action Recognition from Various Data Modalities: A Review. arXiv 2020, arXiv:2012.11866.

26. Liu, B.; Cai, H.; Ju, Z.; Liu, H. RGB-D sensing based human action and interaction analysis: A survey. Pattern Recognit. 2019, 94, 1-12. [CrossRef]

27. Singh, R.; Sonawane, A.; Srivastava, R. Recent evolution of modern datasets for human activity recognition: A deep survey. Multimed. Syst. 2019, 26, 1-24. [CrossRef]

28. Presti, L.L.; La Cascia, M. 3D skeleton-based human action classification: A survey. Pattern Recognit. 2016, 53, 130-147. [CrossRef] 
29. Sedmidubsky, J.; Elias, P.; Budikova, P.; Zezula, P. Content-based Management of Human Motion Data: Survey and Challenges. IEEE Access 2021, 9, 64241-64255. [CrossRef]

30. Rosin, P.L.; Lai, Y.K.; Shao, L.; Liu, Y. RGB-D Image Analysis and Processing; Springer: Berlin/Heidelberg, Germany, 2019.

31. Liu, J.; Shahroudy, A.; Perez, M.L.; Wang, G.; Duan, L.Y.; Kot Chichung, A. NTU RGB + D 120: A Large-Scale Benchmark for 3D Human Activity Understanding. IEEE Trans. Pattern Anal. Mach. Intell. TPAMI 2019, 2684-2701. [CrossRef]

32. Tölgyessy, M.; Dekan, M.; Chovanec, L.; Hubinskỳ, P. Evaluation of the Azure Kinect and Its Comparison to Kinect V1 and Kinect V2. Sensors 2021, 21, 413. [CrossRef]

33. Microsoft. Buy the Azure Kinect Developer kit-Microsoft. 2019. Available online: https://www.microsoft.com/en-us/d/azurekinect-dk/8pp5vxmd9nhq (accessed on 14 June 2021).

34. EB Games. Kinect for Xbox One (Preowned)-Xbox One-EB Games Australia. 2015. Available online: https://www.ebgames.com. au/product/xbox-one/202155-kinect-for-xbox-one-preowned (accessed on 14 June 2021).

35. EB Games. Kinect for Xbox 360 without AC Adapter (Preowned)-Xbox 360-EB Games Australia. 2013. Available online: https:/ / www.ebgames.com.au/product/xbox360/151784-kinect-for-xbox-360-without-ac-adapter-preowned (accessed on 14 June 2021).

36. Intel Corporation. LiDAR Camera L515 \&ndash; Intel ${ }^{\circledR}$ RealSense ${ }^{\mathrm{TM}}$ Depth and Tracking Cameras. 2019. Available online: https: / / www.intelrealsense.com/lidar-camera-1515/(accessed on 14 June 2021).

37. Orbbec 3D. Astra Series-Orbbec. 2021. Available online: https:/ / orbbec3d.com/product-astra-pro (accessed on 14 June 2021).

38. Lee, I.J. Kinect-for-windows with augmented reality in an interactive roleplay system for children with an autism spectrum disorder. Interact. Learn. Environ. 2020, 1-17 . [CrossRef]

39. Yukselturk, E.; Altıok, S.; Başer, Z. Using game-based learning with kinect technology in foreign language education course. J. Educ. Technol. Soc. 2018, 21, 159-173.

40. Pal, M.; Saha, S.; Konar, A. Distance matching based gesture recognition for healthcare using Microsoft's Kinect sensor. In Proceedings of the International Conference on Microelectronics, Computing and Communications (MicroCom), Durga, India, 23-25 January 2016; pp. 1-6.

41. Ketoma, V.K.; Schäfer, P.; Meixner, G. Development and evaluation of a virtual reality grocery shopping application using a multi-Kinect walking-in-place approach. In Proceedings of the International Conference on Intelligent Human Systems Integration, Dubai, UAE, 7-9 January 2018; pp. 368-374.

42. Zhang, Y.; Chen, C.; Wu, Q.; Lu, Q.; Zhang, S.; Zhang, G.; Yang, Y. A Kinect-based approach for 3D pavement surface reconstruction and cracking recognition. IEEE Trans. Intell. Transp. Syst. 2018, 19, 3935-3946. [CrossRef]

43. Keselman, L.; Woodfill, J.I.; Grunnet-Jepsen, A.; Bhowmik, A. Intel(R) RealSense(TM) Stereoscopic Depth Cameras. In Proceedings of the IEEE Conference on Computer Vision and Pattern Recognition (CVPR), Honolulu, HI, USA, 21-26 July 2017; pp. 1267-1276.

44. Drouin, M.A.; Seoud, L. Consumer-Grade RGB-D Cameras. In 3D Imaging, Analysis and Applications; Springer: Berlin/Heidelberg, Germany, 2020; pp. 215-264.

45. Grunnet-Jepsen, A.; Sweetser, J.N.; Woodfill, J. Best Known Methods for Tuning Intel ${ }^{\circledR}$ RealSense $^{\text {TM }}$ Depth Cameras D415. 2018. Available online: https:/ /www.intel.com.au/content/www/au/en/support/articles/000027833/emerging-technologies/intelrealsense-technology.html (accessed on 28 January 2021).

46. Zabatani, A.; Surazhsky, V.; Sperling, E.; Moshe, S.B.; Menashe, O.; Silver, D.H.; Karni, T.; Bronstein, A.M.; Bronstein, M.M.; Kimmel, R. Intel ${ }^{\circledR}$ RealSense ${ }^{\mathrm{TM}}$ SR300 Coded light depth Camera. IEEE Trans. Pattern Anal. Mach. Intell. TPAMI 2019, $2333-2345$. [CrossRef] [PubMed]

47. Coroiu, A.D.C.A.; Coroiu, A. Interchangeability of Kinect and Orbbec Sensors for Gesture Recognition. In Proceedings of the 2018 IEEE 14th International Conference on Intelligent Computer Communication and Processing (ICCP), , Cluj-Napoca, Romania, 6-8 September 2018; pp. 309-315. [CrossRef]

48. Villena-Martínez, V.; Fuster-Guilló, A.; Azorín-López, J.; Saval-Calvo, M.; Mora-Pascual, J.; Garcia-Rodriguez, J.; Garcia-Garcia, A. A Quantitative Comparison of Calibration Methods for RGB-D Sensors Using Different Technologies. Sensors 2017, 17. [CrossRef] [PubMed]

49. Oreifej, O.; Liu, Z. HON4D: Histogram of Oriented 4D Normals for Activity Recognition from Depth Sequences. In Proceedings of the IEEE Conference on Computer Vision and Pattern Recognition (CVPR), Portland, OR, USA, 23-28 June 2013 ; pp. 716-723.

50. Chen, C.; Liu, K.; Kehtarnavaz, N. Real-time Human Action Recognition Based on Depth Motion Maps. J. Real Time Image Process. 2016, 12, 155-163. [CrossRef]

51. Yang, X.; Tian, Y. Effective 3D Action Recognition using EigenJoints. J. Vis. Commun. Image Represent. 2014, 25, 2-11. [CrossRef]

52. Li, M.; Leung, H.; Shum, H.P. Human Action Recognition via Skeletal and Depth based Feature Fusion. In Proceedings of the 9th International Conference on Motion in Games, Burlingame, CA, USA, 10-12 October 2016; pp. 123-132.

53. Yang, X.; Tian, Y. Super Normal Vector for Activity Recognition using Depth Sequences. In Proceedings of the 2014 IEEE Conference on Computer Vision and Pattern Recognition, Columbus, OR, USA, 23-28 June 2014; pp. 804-811.

54. Rahmani, H.; Mahmood, A.; Huynh, D.Q.; Mian, A. Real Time Action Recognition using Histograms of Depth Gradients and Random Decision Forests. In Proceedings of the IEEE Winter Conference on Applications of Computer Vision, (WCACV), SteamBoats Springs, CO, USA, 24-26 March 2014; pp. 626-633.

55. Yang, X.; Zhang, C.; Tian, Y. Recognizing Actions using Depth Motion Maps-based Histograms of Oriented Gradients. In Proceedings of the 20th ACM International Conference on Multimedia, Nara, Japan, 29 October-2 November 2012; pp. 1057-1060. 
56. Chen, C.; Jafari, R.; Kehtarnavaz, N. Action Recognition from Depth Sequences using Depth Motion Maps-based Local Binary Patterns. In Proceedings of the IEEE Winter Conference on Applications of Computer Vision (WCACV), Waikola, HI, USA, 5-9 January 2015; pp. 1092-1099.

57. Chen, W.; Guo, G. TriViews: A General Framework to use 3D Depth Data Effectively for Action Recognition. J. Vis. Commun. Image Represent. 2015, 26, 182-191. [CrossRef]

58. Miao, J.; Jia, X.; Mathew, R.; Xu, X.; Taubman, D.; Qing, C. Efficient Action Recognition from Compressed Depth Maps. In Proceedings of the IEEE International Conference on Image Processing (ICIP), Phoenix, AZ, USA, 25-28 September 2016; pp. 16-20.

59. Xia, L.; Chen, C.; Aggarwal, J. View Invariant Human Action Recognition using Histograms of 3D Joints. In Proceedings of the IEEE Computer Society Conference on Computer Vision and Pattern Recognition (CVPR), Providence, RI, USA, 16-21 June 2012; pp. 20-27.

60. Gowayyed, M.A.; Torki, M.; Hussein, M.E.; El-Saban, M. Histogram of Oriented Displacements (HOD): Describing Trajectories of Human Joints for Action Recognition. In Proceedings of the 23rd International Joint Conference on Artificial Intelligence, Beijing, China, 3-9 August 2013.

61. Pazhoumand-Dar, H.; Lam, C.P.; Masek, M. Joint Movement Similarities for Robust 3D Action Recognition using Skeletal Data. J. Vis. Commun. Image Represent. 2015, 30, 10-21. [CrossRef]

62. Papadopoulos, G.T.; Axenopoulos, A.; Daras, P. Real-time Skeleton-tracking-based Human Action Recognition using Kinect Data. In Proceedings of the International Conference on Multimedia Modeling, Dublin, Ireland, 6-10 January $2014 ;$ pp. $473-483$.

63. Chaaraoui, A.; Padilla-Lopez, J.; Flórez-Revuelta, F. Fusion of Skeletal and Silhouette-based Features for Human Action Recognition with RGB-D Devices. In Proceedings of the IEEE International Conference on Computer Vision (ICCV), Sydney, Australia, 1-8 December 2013; pp. 91-97.

64. Althloothi, S.; Mahoor, M.H.; Zhang, X.; Voyles, R.M. Human Activity Recognition using Multi-features and Multiple Kernel Learning. Pattern Recognit. 2014, 47, 1800-1812. [CrossRef]

65. Liu, L.; Shao, L. Learning Discriminative Representations from RGB-D Video Data. In Proceedings of the International Joint Conference on Artificial Intelligence, Beijing, China, 3-9 August 2013; pp. 1493-1500.

66. Jalal, A.; Kim, Y.H.; Kim, Y.J.; Kamal, S.; Kim, D. Robust Human Activity Recognition from Depth Video using Spatiotemporal Multi-fused Features. Pattern Recognit. 2017, 61, 295-308. [CrossRef]

67. Ni, B.; Pei, Y.; Moulin, P.; Yan, S. Multilevel Depth and Image Fusion for Human Activity Detection. IEEE Trans. Syst. Man Cybern. 2013, 43, 1383-1394.

68. Kong, Y.; Fu, Y. Discriminative relational representation learning for RGB-D action recognition. IEEE Trans. Image Process. 2016, 25, 2856-2865. [CrossRef]

69. Yu, M.; Liu, L.; Shao, L. Structure-preserving binary representations for RGB-D action recognition. IEEE Trans. Pattern Anal. Mach. Intell. 2015, 38, 1651-1664. [CrossRef]

70. Guo, Y.; Liu, Y.; Oerlemans, A.; Lao, S.; Wu, S.; Lew, M.S. Deep learning for visual understanding: A review. Neurocomputing 2016, 187, 27-48. [CrossRef]

71. Yan, S.; Xiong, Y.; Lin, D. Spatial temporal graph convolutional networks for skeleton-based action recognition. In Proceedings of the AAAI Conference on Artificial Intelligence, New Orleans, LA, USA, 2-7 February 2018; Volume 32.

72. Miikkulainen, R.; Liang, J.; Meyerson, E.; Rawal, A.; Fink, D.; Francon, O.; Raju, B.; Shahrzad, H.; Navruzyan, A.; Duffy, N.; et al. Chapter 15-Evolving Deep Neural Networks. In Artificial Intelligence in the Age of Neural Networks and Brain Computing; Academic Press: Cambridge, MA, USA, 2019; pp. 293-312.

73. Hochreiter, S.; Schmidhuber, J. Long short-term memory. Neural Comput. 1997, 9, 1735-1780. [CrossRef]

74. Ronao, C.A.; Cho, S.B. Human activity recognition with smartphone sensors using deep learning neural networks. Expert Syst. Appl. 2016, 59, 235-244. [CrossRef]

75. Zhu, W.; Lan, C.; Xing, J.; Zeng, W.; Li, Y.; Shen, L.; Xie, X. Co-occurrence feature learning for skeleton based action recognition using regularized deep LSTM networks. In Proceedings of the AAAI Conference on Artificial Intelligence, Phoenix, AZ, USA, 12-17 February 2016; Volume 30.

76. Kipf, T.N.; Welling, M. Semi-Supervised Classification with Graph Convolutional Networks. arXiv 2017, arXiv:cs.LG/1609.02907.

77. Caetano, C.; Sena de Souza, J.; Santos, J.; Schwartz, W. SkeleMotion: A New Representation of Skeleton Joint Sequences Based on Motion Information for 3D Action Recognition. In Proceedings of the IEEE International Conference on Advanced Video and Signal Based Surveillance (AVSS), Taipei, Taiwan, 18-21 September 2019; pp. 1-8.

78. Li, W.; Zhang, Z.; Liu, Z. Action recognition based on a bag of 3d points. In Proceedings of the 2010 IEEE Computer Society Conference on Computer Vision and Pattern Recognition, 13-18 June 2010; pp. 9-14.

79. Damen, D.; Doughty, H.; Farinella, G.M.; Fidler, S.; Furnari, A.; Kazakos, E.; Moltisanti, D.; Munro, J.; Perrett, T.; Price, W.; et al. Scaling egocentric vision: The epic-kitchens dataset. In Proceedings of the European Conference on Computer Vision (ECCV), Munich, Germany, 8-14 September 2018; pp. 720-736.

80. Soomro, K.; Zamir, A.R.; Shah, M. UCF101: A Dataset of 101 Human Actions Classes From Videos in The Wild. arXiv 2012, arXiv:cs.CV/1212.0402. 
81. Das, S.; Dai, R.; Koperski, M.; Minciullo, L.; Garattoni, L.; Bremond, F.; Francesca, G. Toyota Smarthome: Real-World Activities of Daily Living. In Proceedings of the Internation Conference on Computer Vision (ICCV), Seoul, Korea, 27 October-2 November 2019.

82. Ni, B.; Wang, G.; Moulin, P. RGBD-HuDaAct: A color-depth video database for human daily activity recognition. In Proceedings of the Internation Conference on Computer Vision (ICCV), Barcelona, Spain, 6-13 November 2011; pp. 1147-1153.

83. Chen, C.; Jafari, R.; Kehtarnavaz, N. UTD-MHAD: A Multimodal Dataset for Human Action Recognition Utilizing A Depth Camera and A Wearable Inertial Sensor. In Proceedings of the Int. Conf. on Image Processing, Quebec City, QC, Canada, 27-30 September 2015; pp. 168-172.

84. Sigurdsson, G.A.; Varol, G.; Wang, X.; Farhadi, A.; Laptev, I.; Gupta, A. Hollywood in Homes: Crowdsourcing Data Collection for Activity Understanding. In Proceedings of the European Conference Computer Vision (ECCV), Amsterdam, The Netherlands, 11-14 October 2016; pp. 510-526.

85. Korbar, B.; Tran, D.; Torresani, L. SCSampler: Sampling Salient Clips from Video for Efficient Action Recognition. In Proceedings of the International Conference on Compututer Vision (ICCV), Seoul, Korea, 27 October-2 November 2019; pp. 6231-6241.

86. Karpathy, A.; Toderici, G.; Shetty, S.; Leung, T.; Sukthankar, R.; Fei-Fei, L. Large-scale Video Classification With Convolutional Neural Networks. In Proceedings of the IEEE Computer Society Conference Computer Vision Pattern Recognit. (CVPR), Columbus, OR, USA, 24-27 June 2014; pp. 1725-1732.

87. Kim, S.; Yun, K.; Park, J.; Choi, J. Skeleton-Based Action Recognition of People Handling Objects. In Proceedings of the IEEE Winter Conference on Applications of Computer Vision (WCACV), Waikoloa, HI, USA, 7-11 January 2019; pp. 61-70.

88. Zhu, J.; Zou, W.; Xu, L.; Hu, Y.; Zhu, Z.; Chang, M.; Huang, J.; Huang, G.; Du, D. Action Machine: Rethinking Action Recognition in Trimmed Videos. arXiv 2018, arXiv:cs.CV/1812.05770.

89. Kuehne, H.; Jhuang, H.; Garrote, E.; Poggio, T.; Serre, T. HMDB: A Large Video Database for Human Motion Recognition. In Proceedings of the International Conference on Computer Vision (ICCV), Barcelona, Spain, 6-13 November 2011; pp. $2556-2563$.

90. Shahroudy, A.; Liu, J.; Ng, T.T.; Wang, G. NTU RGB + D: A Large Scale Dataset for 3D Human Activity Analysis. In Proceedings of the IEEE Computer Society Conference Computer Vision Pattern Recognition (CVPR), Los Alamitos, CA, USA, 27-30 June 2016; pp. 1010-1019.

91. Wang, J.; Nie, X.; Xia, Y.; Wu, Y.; Zhu, S.C. Cross-view Action Modeling, Learning and Recognition. In Proceedings of the IEEE Computer Society Conference Computer Vision Pattern Recognition (CVPR), Columbus, OH, USA, 23-28 June 2014; pp. 2649-2656.

92. Zhao, Y.; Liu, Z.; Yang, L.; Cheng, H. Combing RGB and Depth Map Features for human activity recognition. In Proceedings of the Asia Pacific Signal and Information Processing Association Annual Summit and Conference, Hollywood, CA, USA, 3-6 December 2012; pp. 1-4.

93. Ye, J.; Li, K.; Qi, G.J.; Hua, K.A. Temporal order-preserving dynamic quantization for human action recognition from multimodal sensor streams. In Proceedings of the 5th ACM on International Conference on Multimedia Retrieval, Shanghai, China, 23-26 June 2015; pp. 99-106.

94. Shahroudy, A.; Ng, T.T.; Gong, Y.; Wang, G. Deep multimodal feature analysis for action recognition in RGB + D videos. IEEE Trans. Pattern Anal. Mach. Intell. TPAMI 2017, 40, 1045-1058. [CrossRef] [PubMed]

95. Ryoo, M.S.; Piergiovanni, A.; Tan, M.; Angelova, A. AssembleNet: Searching for Multi-Stream Neural Connectivity in Video Architectures. arXiv 2020, arXiv:cs.CV/1905.13209.

96. Tran, D.; Wang, H.; Torresani, L.; Feiszli, M. Video Classification with Channel-separated Convolutional Networks. In Proceedings of the International Conference on Computer Vision (ICCV), Seoul, Korea, 27 October-2 November 2019; pp. 5552-5561.

97. Wang, L.; Koniusz, P.; Huynh, D.Q. Hallucinating iDT Descriptors and i3D Optical Flow Features for Action Recognition with CNNs. In Proceedings of the International Conference on Computer Vision (ICCV), Seoul, Korea, 27 October-2 November 2019; pp. 8698-8708.

98. Kazakos, E.; Nagrani, A.; Zisserman, A.; Damen, D. EPIC-Fusion: Audio-Visual Temporal Binding for Egocentric Action Recognition. In Proceedings of the IEEE/CVF International Conference on Computer Vision (ICCV), Seoul, Korea, 27 October-2 November 2019.

99. Das, S.; Sharma, S.; Dai, R.; Brémond, F.; Thonnat, M. VPN: Learning Video-Pose Embedding for Activities of Daily Living. In ECCV 2020; Springer: Cham, Switzerland, 2020; pp. 72-90.

100. Islam, M.M.; Iqbal, T. HAMLET: A Hierarchical Multimodal Attention-based Human Activity Recognition Algorithm. arXiv 2020, arXiv:cs.RO/2008.01148.

101. Davoodikakhki, M.; Yin, K. Hierarchical action classification with network pruning. In International Symposium on Visual Computing; Springer: Berlin/Heidelberg, Germany, 2020; pp. 291-305.

102. Wang, P.; Li, W.; Gao, Z.; Zhang, J.; Tang, C.; Ogunbona, P. Deep Convolutional Neural Networks for Action Recognition Using Depth Map Sequences. arXiv 2015, arXiv:cs.CV/1501.04686.

103. Wang, P.; Wang, S.; Gao, Z.; Hou, Y.; Li, W. Structured Images for RGB-D Action Recognition. In Proceedings of the 2017 IEEE International Conference on Computer Vision (ICCV 2017), Venice, Italy, 22-29 October 2017; pp. 1005-1014.

104. Song, S.; Lan, C.; Xing, J.; Zeng, W.; Liu, J. Spatio-Temporal Attention-Based LSTM Networks for 3D Action Recognition and Detection. IEEE Trans. Image Process. TIP 2018, 27, 3459-3471. [CrossRef] 
105. Ye, Y.; Tian, Y. Embedding Sequential Information into Spatiotemporal Features for Action Recognition. In Proceedings of the 2016 IEEE Conference on Computer Vision and Pattern Recognition (CVPR), Las Vegas, NV, USA, 27-30 June 2016; pp. 1110-1118.

106. Krizhevsky, A.; Sutskever, I.; Hinton, G.E. ImageNet Classification with Deep Convolutional Neural Networks. In Proceedings of the Advances in Neural Information Processing Systems; Curran Associates, Inc.: Lake Tahoe, CA, USA, 2012; pp. 1097-1105.

107. Baccouche, M.; Mamalet, F.; Wolf, C.; Garcia, C.; Baskurt, A. Sequential Deep Learning for Human Action Recognition. In International Workshop on Human Behavior Understanding; Springer: Berlin/Heidelberg, Germany, 2011; pp. 29-39.

108. Ji, S.; Xu, W.; Yang, M.; Yu, K. 3D Convolutional Neural Networks for Human Action Recognition. IEEE Trans. Pattern Anal. Mach. Intell. TPAMI 2013, 35, 221-231. [CrossRef]

109. Simonyan, K.; Zisserman, A. Two-stream Convolutional Networks for Action Recognition in Videos. In Proceedings of the 27th International Conference on Neural Information Processing Systems, Montreal, QC, Canada, 8-13 December 2014 ; pp. 568-576.

110. Zhang, B.; Wang, L.; Wang, Z.; Qiao, Y.; Wang, H. Real-Time Action Recognition With Deeply Transferred Motion Vector CNNs. IEEE Trans. Image Process. TIP 2018, 27, 2326-2339. [CrossRef] [PubMed]

111. Feichtenhofer, C.; Pinz, A.; Zisserman, A. Convolutional Two-Stream Network Fusion for Video Action Recognition. In Proceedings of the IEEE Computer Society Conference Computer Vision Pattern Recognition (CVPR), Las Vegas, NV, USA, 27-30 June 2016.

112. Wang, L.; Xiong, Y.; Wang, Z.; Qiao, Y.; Lin, D.; Tang, X.; Gool, L.V. Temporal Segment Networks: Towards Good Practices for Deep Action Recognition. In Proceedings of the European Conference on Computer Vision (ECCV), Amsterdam, The Netherlands, 11-14 October 2016; pp. 20-36.

113. Lan, Z.; Zhu, Y.; Hauptmann, A.G.; Newsam, S. Deep Local Video Feature for Action Recognition. In Proceedings of the IEEE Computer Society Conference Computer Vision Pattern Recognition (CVPR), Honolulu, HI, USA, 21-26 July 2017; pp. 1219-1225.

114. Zhou, B.; Andonian, A.; Torralba, A. Temporal Relational Reasoning in Videos. In Proceedings of the European Conference on Computer Vision (ECCV), Munich, Germany, 8-14 September 2018; Volume 11205, pp. 831-846.

115. Carreira, J.; Zisserman, A. Quo Vadis, Action Recognition? A New Model and the Kinetics Dataset. In Proceedings of the IEEE Computer Society Conference Computer Vision Pattern Recognit. (CVPR), Honolulu, HI, USA, 21-26 July 2017 ; pp. $4724-4733$.

116. Zhu, Y.; Lan, Z.; Newsam, S.; Hauptmann, A. Hidden Two-Stream Convolutional Networks for Action Recognition. In Proceedings of the Asian Conference on Computer Vision, Perth, Australia, 2-6 December 2018; pp. 363-378.

117. Ng, J.Y.H.; Hausknecht, M.; Vijayanarasimhan, S.; Vinyals, O.; Monga, R.; Toderici, G. Beyond Short Snippets: Deep Networks for Video Classification. In Proceedings of the IIEEE Conference on Computer Vision and Pattern Recognition (CVPR), Boston, MA, USA, 7-12 June 2015; pp. 4694-4702.

118. Qiu, Z.; Yao, T.; Mei, T. Learning Spatio-Temporal Representation with Pseudo-3D Residual Networks. In Proceedings of the International Conference on Computer Vision (ICCV), Venice, Italy, 22-29 October 2017; pp. 5533-5541.

119. Liu, J.; Shahroudy, A.; Xu, D.; Kot, A.C.; Wang, G. Skeleton-Based Action Recognition Using Spatio-Temporal LSTM Network with Trust Gates. IEEE Trans. Pattern Anal. Mach. Intell. TPAMI 2018, 40, 3007-3021. [CrossRef] [PubMed]

120. Donahue, J.; Hendricks, L.A.; Rohrbach, M.; Venugopalan, S.; Guadarrama, S.; Saenko, K.; Darrell, T. Long-Term Recurrent Convolutional Networks for Visual Recognition and Description. IEEE Trans. Pattern Anal. Mach. Intell. TPAMI 2017, 39, 677-691. [CrossRef]

121. Abu-El-Haija, S.; Kothari, N.; Lee, J.; Natsev, P.; Toderici, G.; Varadarajan, B.; Vijayanarasimhan, S. YouTube-8M: A Large-Scale Video Classification Benchmark. arXiv 2016, arXiv:cs.CV/1609.08675.

122. Caba Heilbron, F.; Victor Escorcia, B.G.; Niebles, J.C. ActivityNet: A Large-Scale Video Benchmark for Human Activity Understanding. In Proceedings of the IEEE Computer Society Conference Computer Vision Pattern Recognition (CVPR), Boston, MA, USA, 7-12 June 2015; pp. 961-970.

123. Moon, J.; Jin, J.; Kwon, Y.; Kang, K.; Park, J.; Park, K. Extensible Hierarchical Method of Detecting Interactive Actions for Video Understanding. ETRI J. 2017, 39, 502-513. [CrossRef]

124. Moon, J.; Kwon, Y.; Kang, K.; Park, J. ActionNet-VE Dataset: A Dataset for Describing Visual Events by Extending VIRAT Ground 2.0. In Proceedings of the 8th International Conference on Signal Processing, Image Processing and Pattern Recognition (SIP), Jeju, Korea, 25-28 November 2015; pp. 1-4.

125. Liu, Y.; Ma, L.; Zhang, Y.; Liu, W.; Chang, S. Multi-Granularity Generator for Temporal Action Proposal. In Proceedings of the IEEE Computer Society Conference Computer Vision Pattern Recognition (CVPR), Long Beach, CA, USA, 15-20 June 2019; pp. 3604-3613.

126. Qiu, Z.; Yao, T.; Ngo, C.W.; Tian, X.; Mei, T. Learning Spatio-Temporal Representation With Local and Global Diffusion. In Proceedings of the IEEE Computer Society Conference Computer Vision Pattern Recognition (CVPR), Long Beach, CA, USA, 15-20 June 2019; pp. 12056-12065.

127. Lin, J.; Gan, C.; Han, S. TSM: Temporal Shift Module for Efficient Video Understanding. In Proceedings of the International Conference on Computer Vision (ICCV), Seoul, Korea, 27 October-2 November 2019; pp. 7083-7093.

128. Girdhar, R.; João Carreira, J.; Doersch, C.; Zisserman, A. Video Action Transformer Network. In Proceedings of the IEEE Computer Society Conference Computer Vision Pattern Recognition (CVPR), Long Beach, CA, USA, 15-20 June 2019 ; pp. 244-253.

129. Hu, J.F.; Zheng, W.S.; Pan, J.; Lai, J.; Zhang, J. Deep Bilinear Learning for RGB-D Action Recognition. In Proceedings of the European Conference Computer Vision (ECCV), Munich, Germany, 8-14 September 2018; pp. 346-362. 
130. Sudhakaran, S.; Escalera, S.; Lanz, O. Gate-Shift Networks for Video Action Recognition. In Proceedings of the IEEE Computer Society Conference Computer Vision Pattern Recognition (CVPR), Los Alamitos, CA, USA, 26 June-1 July 2020; pp. 1099-1108.

131. Liu, X.; Lee, J.; Jin, H. Learning Video Representations From Correspondence Proposals. In Proceedings of the IEEE Computer Society Conference Computer Vision Pattern Recognition (CVPR), Long Beach, CA, USA, 15-20 June 2019; pp. 4273-4281.

132. Materzynska, J.; Berger, G.; Bax, I.; Memisevic, R. The Jester Dataset: A Large-Scale Video Dataset of Human Gestures. In Proceedings of the International Conference on Computer Vision, Seoul, Korea, 27-28 October 2019.

133. Martin, M.; Roitberg, A.; Haurilet, M.; Horne, M.; Reiß, S.; Voit, M.; Stiefelhagen, R. Drive \& Act: A Multimodal Dataset for Fine-Grained Driver Behavior Recognition in Autonomous Vehicles. In Proceedings of the International Conference on Computer Vision (ICCV), Seoul, Korea, 27 October-2 November 2019; pp. 2801-2810.

134. Munro, J.; Damen, D. Multi-modal Domain Adaptation for Fine-grained Action Recognition. In Proceedings of the IEEE Computer Society Conference Computer Vision Pattern Recognition (CVPR), Seattle, WA, USA, 14-19 June 2020.

135. Jiang, H.; Li, Y.; Song, S.; Liu, J. Rethinking Fusion Baselines for Multimodal Human Action Recognition. In Proceedings of the 19th Pacific-Rim Conference on Multimedia, Advances in Multimedia Information Processing, Hefei, China, 21-22 September 2018; pp. 178-187.

136. Shereena, V.; David, J.M. Content based image retrieval: Classification using neural networks. Int. J. Multimed. Its Appl. 2014, 6,31 .

137. Bhaumik, H.; Bhattacharyya, S.; Nath, M.D.; Chakraborty, S. Real-time storyboard generation in videos using a probability distribution based threshold. In Proceedings of the Fifth International Conference on Communication Systems and Network Technologies, Gwalior, India 4-6 April 2015; pp. 425-431.

138. Lim, J.H.; Teh, E.Y.; Geh, M.H.; Lim, C.H. Automated classroom monitoring with connected visioning system. In Proceedings of the 2017 Asia-Pacific Signal and Information Processing Association Annual Summit and Conference (APSIPA ASC), Kuala Lumpur, Malaysia, 12-15 December 2017; pp. 386-393.

139. Arifoglu, D.; Bouchachia, A. Activity recognition and abnormal behaviour detection with recurrent neural networks. Procedia Comput. Sci. 2017, 110, 86-93. [CrossRef]

140. You, I.; Choo, K.K.R.; Ho, C.L. A smartphone-based wearable sensors for monitoring real-time physiological data. Comput. Electr Eng. 2018, 65, 376-392.

141. Laptev, I.; Marszalek, M.; Schmid, C.; Rozenfeld, B. Learning realistic human actions from movies. In Proceedings of the 2008 IEEE Conference on Computer Vision and Pattern Recognition, Anchorage, AK, USA, 23-28 June 2008; pp. 1-8.

142. Wang, P.; Cao, Y.; Shen, C.; Liu, L.; Shen, H.T. Temporal pyramid pooling-based convolutional neural network for action recognition. IEEE Trans. Circuits Syst. Video Technol. 2016, 27, 2613-2622. [CrossRef]

143. Kumar, K.; Kishore, P.; Kumar, D.A.; Kumar, E.K. Indian classical dance action identification using adaboost multiclass classifier on multifeature fusion. In Proceedings of the 2018 Conference on Signal Processing And Communication Engineering Systems (SPACES), Vijayawada, India, 4-5 January 2018; pp. 167-170.

144. Castro, D.; Hickson, S.; Sangkloy, P.; Mittal, B.; Dai, S.; Hays, J.; Essa, I. Let's Dance: Learning from Online Dance Videos. arXiv 2018, arXiv:1801.07388.

145. Feng, Y.; Yuan, Y.; Lu, X. Learning deep event models for crowd anomaly detection. Neurocomputing 2017, 219, 548-556. [CrossRef]

146. Li, T.; Liu, J.; Zhang, W.; Ni, Y.; Wang, W.; Li, Z. UAV-Human: A Large Benchmark for Human Behavior Understanding with Unmanned Aerial Vehicles. arXiv 2021, arXiv:2104.00946.

147. Thomas, G.; Gade, R.; Moeslund, T.B.; Carr, P.; Hilton, A. Computer vision for sports: Current applications and research topics. Comput. Vis. Image Underst. 2017, 159, 3-18. [CrossRef]

148. Ullah, A.; Muhammad, K.; Haq, I.U.; Baik, S.W. Action recognition using optimized deep autoencoder and CNN for surveillance data streams of non-stationary environments. Future Gener. Comput. Syst. 2019, 96, 386-397. [CrossRef]

149. Qi, M.; Wang, Y.; Qin, J.; Li, A.; Luo, J.; Van Gool, L. stagNet: An attentive semantic RNN for group activity and individual action recognition. IEEE Trans. Circuits Syst. Video Technol. 2019, 30, 549-565. [CrossRef]

150. Gao, F.; Wang, C.; Li, C. A Combined Object Detection Method With Application to Pedestrian Detection. IEEE Access 2020, 8, 194457-194465. [CrossRef] 